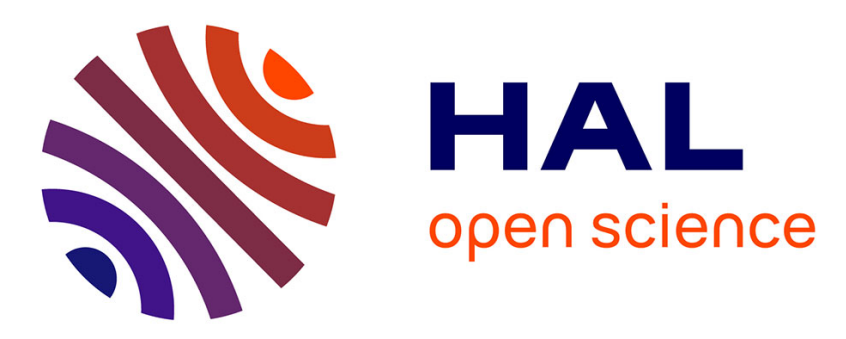

\title{
Statistical theory of designed quantum transport across disordered networks
}

Mattia Walschaers, Roberto Mulet, Thomas Wellens, Andreas Buchleitner

\section{To cite this version:}

Mattia Walschaers, Roberto Mulet, Thomas Wellens, Andreas Buchleitner. Statistical theory of designed quantum transport across disordered networks. Physical Review E: Statistical, Nonlinear, and Soft Matter Physics, 2015, 91 (4), 10.1103/PhysRevE.91.042137 . hal-03216943

\section{HAL Id: hal-03216943 \\ https://hal.science/hal-03216943}

Submitted on 4 May 2021

HAL is a multi-disciplinary open access archive for the deposit and dissemination of scientific research documents, whether they are published or not. The documents may come from teaching and research institutions in France or abroad, or from public or private research centers.
L'archive ouverte pluridisciplinaire HAL, est destinée au dépôt et à la diffusion de documents scientifiques de niveau recherche, publiés ou non, émanant des établissements d'enseignement et de recherche français ou étrangers, des laboratoires publics ou privés. 


\title{
Statistical theory of designed quantum transport across disordered networks
}

\author{
Mattia Walschaers ${ }^{1,2, *}$ Roberto Mulet, ${ }^{1,3, \dagger}$ Thomas Wellens, ${ }^{1, \ddagger}$ and Andreas Buchleitner ${ }^{1,4, \S}$ \\ ${ }^{1}$ Physikalisches Institut, Albert-Ludwigs-Universität Freiburg, Hermann-Herder-Str. 3, D-79104 Freiburg, Germany \\ ${ }^{2}$ Instituut voor Theoretische Fysica, University of Leuven, Celestijnenlaan 200D, B-3001 Heverlee, Belgium \\ ${ }^{3}$ Complex Systems Group, Department of Theoretical Physics, University of Havana, Cuba \\ ${ }^{4}$ Freiburg Institute for Advanced Studies, Albert-Ludwigs-Universität Freiburg, Albertstr. 19, D-79104 Freiburg, Germany
}

(Received 9 September 2014; published 28 April 2015)

\begin{abstract}
We explain how centrosymmetry, together with a dominant doublet of energy eigenstates in the local density of states, can guarantee interference-assisted, strongly enhanced, strictly coherent quantum excitation transport between two predefined sites of a random network of two-level systems. Starting from a generalization of the chaos-assisted tunnelling mechanism, we formulate a random matrix theoretical framework for the analytical prediction of the transfer time distribution, of lower bounds of the transfer efficiency, and of the scaling behavior of characteristic statistical properties with the size of the network. We show that these analytical predictions compare well to numerical simulations, using Hamiltonians sampled from the Gaussian orthogonal ensemble.
\end{abstract}

DOI: 10.1103/PhysRevE.91.042137

PACS number(s): 05.60.Gg, 03.65.Xp, 72.10.-d, 82.20.Xr

\section{INTRODUCTION}

The impact of quantum interference effects on transport phenomena defines a multi-facetted area of research, with a wide range of incarnations in condensed [1] and soft matter [2], mesoscopic physics [3], quantum chaos [4,5], quantum computing [6,7], light-matter interaction [8-14], and, rather recently, photobiology [15-20]. However, the deterministic control of quantum interference contributions to transport is rightfully considered a subtle problem which turns ever more difficult with an increasing density of states, since this implies that more and more relative phases need to be carefully controlled. Any uncontrolled perturbation of these then has potentially very detrimental effects on the control target (much as in a misaligned Fabry-Pérot cavity) [21]. This is why quantum engineers traditionally dislike noise and disorder, generally invoking strong symmetry properties (such as the translational invariance of a lattice) to guarantee that the desired quantum effects prevail. Of course, as the system size is scaled up, and almost unavoidably so its complexity, perturbations of such symmetries get ever more likely.

On the other hand, it has long been known in solid state and statistical physics that quantum interference effects can actually induce very strong signatures on the statistics of characteristic transport coefficients, even in the presence of strong disorder, Anderson localization arguably being the most prominent example [13,22,23]. More recently, it therefore emerges in diverse areas that disorder may actually be conceived as a robust handle of (statistical rather than deterministic) quantum control [5,18,24-27], in particular on scales which preclude deterministic control on a microscopic level.

One possible, specific scenario for such statistical quantum control is motivated by the ever more consolidating experimental evidence for nontrivial, long-lasting quantum coherence in the strongly optimised excitation transport in photosynthetic

\footnotetext{
*mattia@itf.fys.kuleuven.be

†roberto.mulet@gmail.com

†thomas.wellens@physik.uni-freiburg.de

§a.buchleitner@physik.uni-freiburg.de
}

light harvesting complexes of plants and bacteria [15-17]. These supramolecular and hierarchically structured objects come in rather variable architectures for different biological species, but all share the functional purpose of transporting energy to some reaction center where the plant chemistry is initiated. Ideally, this energy transport should occur with minimal loss, and that might be an evolutionary incentive for also rapid transport. Yet, irrespective of their specific, coarse-grained architectures, all these complexes are garnished by some level of disorder, i.e., their different realizations in the same biological organism exhibit modifications on the microscopic level, simply as a consequence of the enormous complexity of the larger biological structure they are part of. Therefore, the experimentally documented efficiency (close to 100\%) of the excitation transport unavoidably implies a disorder average, $\left\langle e^{-i t H}\right\rangle_{\text {disorder }}$ (where $H$ is the Hamiltonian), and tells us that nature found a way to guarantee near-to-deterministic delivery despite the presence of uncontrolled structural variations on a microscopic level. This stands against a common practice in the literature [28,29], where one uses published Hamiltonian data, e.g., Ref. [30], to describe the coherent backbone dynamics in these molecular complexes: Since these data in general result from (typically spectroscopic) experiments on solutions of such complexes, fluctuations cannot be resolved and an implicit disorder average in the reconstructed Hamiltonian, $\langle H\rangle_{\text {disorder }}$, is always present. The dynamics, however, is not self-averaging, $\left\langle e^{-i t H}\right\rangle_{\text {disorder }} \neq e^{-i t\langle H\rangle_{\text {disorder }} \text {, }}$ and therefore using such average Hamiltonians will typically fail to capture all the relevant physics. The philosophy of our present contribution is exactly to emphasize the potential of disorder-induced statistical effects to optimize relevant transport observables, such as the transfer efficiency, in the presence of quantum interference. Ultimately, such approach may help to identify experimentally implementable methods to certify the quantum or rather classical origin of the observed transfer efficiencies.

We did argue earlier $[18,24,31,32]$ that one possible, and strictly quantum, candidate mechanism leading to large and exceptionally rapid excitation transfer in photosynthetic light harvesting units is constructive multipath quantum interference of the many transmission amplitudes from input to 
output: Reducing the macromolecular complex to a random network, the molecular subunits which constitute the complex are localized at the network's nodes and considered as identical two-level systems with two distinct electronic states, coupled by dipole-dipole interactions. In such strongly simplifying model, the randomness of the network's sites' positions substitutes for the realization-dependent changes of the local environment of the molecular network's constituents and accounts for the uncertainties in the matrix representations of the effective Hamiltonians which can be found in the literature [30]. Even though minimalistic, we argue that this description proves to be qualitatively sufficient in capturing the essential physics which arises due to disorder. Clearly, this approach is inspired by the fundamental idea of random matrix theory (RMT) [33], and strong, quantum interference-induced fluctuations of characteristic transport coefficients are to be expected when sampling over different network realizations. We could show [18] that the statistics of these fluctuations can be efficiently controlled by imposing just two constraints on the otherwise random structure of the network: centrosymmetry and the presence of a dominant doublet in the network's spectrum. With these ingredients, it is indeed possible to make the distribution of transfer efficiencies collapse on a narrow interval very close to unity, and to guarantee rather rapid transfer times, without the need to control the microscopic hardwiring of the network: a clear incident of the above statistical quantum control.

It is the purpose of the present article to spell out the details of the underlying theory, and to scrutinise the scaling properties of the thus "engineered" statistical distributions with the network size, i.e., the number of its elementary molecular sites. Given the generality of the random graph model which we are building on, as well as the ubiquity of disorder or structural perturbations in large networks, we trust that the results here presented do not only provide a fresh perspective for the discussion of quantum effects in photosynthetic light harvesting, but equally much on excitation transport in cold Rydberg gases [34], as well as on quantum walks on random graphs or on robust, quantum walk-based quantum computing design [35-37].

\section{THE MODEL}

Consider a single excitation propagating on a disordered network of $N$ sites. To each site $i$ we associate a quantum state $|i\rangle$ which represents the state where the excitation is fully localized at this very site. These states span the single-excitation Hilbert space of our model. The goal is to transport the excitation from an input site $\mid$ in $\rangle$ to an output site |out $\rangle$ [38]. To mimic disorder, we describe the interaction among the sites by a $N \times N$ Hamiltonian $H$ chosen from the Gaussian Orthogonal Ensemble (GOE) [33], with the additional constraint that the Hamiltonian be centrosymmetric with respect to $\mid$ in $\rangle$ and $\mid$ out $\rangle$. This symmetry is defined by $J H=H J$ and $\mid$ in $\rangle=J \mid$ out $\rangle$, where $J$ is the exchange matrix, $J_{i, j}=\delta_{i, N-j+1}$ [39]. This design principle is motivated by previous results $[32,40]$ suggesting that centrosymmetric Hamiltonians deduced from dipoles randomly distributed within a sphere are statistically more likely to mediate efficient transport than unconstrained random Hamiltonians.
In technical terms, the GOE is characterized by the parameter $\xi$, which describes the density of states as half the radius of Wigner's semicircle [41]. More explicitly, we define our ensemble of interest in terms of a probability distribution on matrix elements given by

$$
H_{i j} \sim \begin{cases}\mathcal{N}\left(0, \frac{2 \xi^{2}}{N}\right) & \text { if } \quad i=j \quad \text { or } \quad i=N-j+1 \\ \mathcal{N}\left(0, \frac{\xi^{2}}{N}\right) & \text { else }\end{cases}
$$

where $\mathcal{N}$ denotes the normal distribution with its mean and variance as first and second argument, respectively. The centrosymmetry constraint practically implies that $H_{i, j}=$ $H_{i, N-j+1}=H_{N-i+1, j}=H_{N-i+1, N-j+1}$ (i.e., the matrix representation of $H$ is invariant under mirroring with respect to the matrix' center), which also guarantees that $E=H_{\text {in, in }}=$ $H_{\text {out,out }}$. The choice of a variance $\xi^{2} / N$ is closely related to the behavior of the spectral density. The specific scaling with $N$ guarantees that the ensemble-averaged density of states is independent of $N$ and is always given by a semicircular distribution of radius $2 \xi$ [41].

Within this ensemble, the input and output sites (and therefore also the associated states) are defined as those that couple the weakest, with coupling $V=\min _{i}\left|H_{i, N-i+1}\right|$. This definition originates from the idea that the input and output are "farthest apart" [what is a suggestive assumption, e.g., when considering the paradigmatic Fenna Matthews Olson (FMO) light harvesting complex as a macromolecular, 3D "wire" which connects the antenna complex to the reaction center] [30]. To avoid the necessity to distinguish between $H_{\text {in,out }}$ and $V$, we will always consider $H_{\text {in,out }}$ to be positive. This boils down to multiplying the full Hamiltonian by -1 if $H_{\text {in,out }}$ is negative for some sampled Hamiltonian. It can be easily verified that this will not cause any problems in the following derivations, yet makes the notation somewhat lighter.

Each of the thus defined Hamiltonians generates a time evolution $|\phi(t)\rangle=\exp (-i t H)|\phi(0)\rangle$ (we set $\hbar \equiv 1$ ) of the initial state $|\phi(0)\rangle=\mid$ in $\rangle$. Focusing on the excitation transfer from |in $\rangle$ to $\mid$ out $\rangle$, a possible measure of the transfer efficiency is

$$
\mathcal{P}_{H}=\max _{t \in\left[0, T_{R}\right)} \mid\left.\langle\text { out }, \phi(t)\rangle\right|^{2},
$$

where $T_{R}$ is the Rabi time, given by $T_{R}=\pi / 2 V[24,31,42]$. This is the time needed for an excitation to be fully transferred from input to output when all sites except for $\mid$ in $\rangle$ and $\mid$ out $\rangle$ are discarded. Therefore, transport can be considered "efficient" if the intermediate sites of the network accelerate the transfer process as compared to the direct coupling between $\mid$ in $\rangle$ and $\mid$ out $\rangle$. We thus set out to identify necessary and/or sufficient conditions for $H$ to be efficient and to render the transport as fast as possible.

\section{A. Centrosymmetry}

We start with a closer scrutiny of the properties of centrosymmetric matrices and emphasize those aspects which are relevant in the context of quantum transport theory. We will explain why centrosymmetry is an important design principle to enhance the excitation transfer and also indicate why this symmetry alone is insufficient to guarantee efficiency in the above sense. 
Given the definition (2) of $\mathcal{P}_{H}$, we are interested in the behavior of $\mid\left.\langle$ out, $\phi(t)\rangle\right|^{2}$. To relate transport properties to the spectral properties of the underlying Hamiltonian, we use the spectral decomposition

$$
\mid\left.\langle\text { out }, \phi(t)\rangle\right|^{2}=\mid\left.\sum_{i=1}^{N} e^{-i t E_{i}}\left\langle\text { out }, \eta_{i}\right\rangle\left\langle\eta_{i}, \text { in }\right\rangle\right|^{2},
$$

where $\eta_{i}$ and $E_{i}$ denote the eigenvectors and eigenvalues of the Hamiltonian $H$, respectively. This expression highlights the eigenvectors' very crucial role for the transport: They determine which sites can be reached from a given input site. If there were no eigenvectors that are significantly localised on both, |in $\rangle$ and $\mid$ out $\rangle$, transport would not be possible. The eigenvalues determine the timescale at which transport occurs.

Centrosymmetry mainly impacts the eigenvectors of the Hamiltonian: It is shown in Ref. [39] that a centrosymmetric matrix also has centrosymmetric eigenvectors. This implies that $J\left|\eta_{i}\right\rangle= \pm\left|\eta_{i}\right\rangle$, where $J$ is the symmetry operator as defined at the beginning of Sec. II. Since we define the Hamiltonian to be centrosymmetric with respect to input and output, we know that, by construction, $J \mid$ out $\rangle=\mid$ in $\rangle$. With the centrosymmetry of the eigenvectors, it follows that $\left\langle\right.$ out, $\left.\eta_{i}\right\rangle\left\langle\eta_{i}\right.$,in $\rangle=$ $\pm \mid\left.\left\langle\right.$ in, $\left.\eta_{i}\right\rangle\right|^{2}= \pm \mid\left.\left\langle\right.$ out,$\left.\eta_{i}\right\rangle\right|^{2}$. Consequently, there is a relation between the probability to have transport from in to out and the return probability. Since we know that, due to weak localization effects, there is always an enhanced return probability $[24,43]$, we expect to find a corresponding effect for the transfer from in to out.

Due to its centrosymmetry, $H$ can be cast, through an orthogonal transformation, into the following block diagonal representation [39] in the eigenbasis of the exchange matrix $J$ :

$$
H=\left(\begin{array}{ll}
H^{+} & 0 \\
0 & H^{-}
\end{array}\right)
$$

Both, $H^{+}$and $H^{-}$, are $N / 2 \times N / 2$ matrices from the GOE. This is a consequence of the block diagonalization [39], combined with the fact that the sum of normally distributed variables is itself a normally distributed variable.

Two eigenvectors of $J$ have the form

$$
\left.\left.| \pm\rangle=\frac{1}{\sqrt{2}}(\mid \text { in }\rangle \pm \mid \text { out }\right\rangle\right)
$$

Using $|+\rangle$ and $|-\rangle$ to express $\mid$ in $\rangle$ and $\mid$ out $\rangle$ allows us to rewrite (2) as

$$
\mathcal{P}_{H}=\max _{t \in\left[0, T_{R}\right)} \frac{1}{4}\left|\left\langle e^{-i t H^{+}}\right\rangle_{+}-\left\langle e^{-i t H^{-}}\right\rangle_{-}\right|^{2} .
$$

The two terms in this expression are statistically independent. Hence, we need to understand the evolution of $|+\rangle$ and $|-\rangle$ under the unitaries generated by $\mathrm{H}^{+}$and $\mathrm{H}^{-}$. In order to do so, we express $\mathcal{P}_{H}$ in terms of the eigenvectors $\left|\eta_{i}^{ \pm}\right\rangle$and of the eigenvalues $E_{i}^{ \pm}$of $H^{ \pm}$:

$\mathcal{P}_{H}=\left.\max _{t \in\left[0, T_{R}\right)} \frac{1}{4}\left|\sum_{i} e^{-i t E_{i}^{+}}\right|\left\langle\eta_{i}^{+},+\right\rangle\right|^{2}-\left.\sum_{i} e^{-i t E_{i}^{-}}\left|\left\langle\eta_{i}^{-},-\right\rangle\right|^{2}\right|^{2}$.

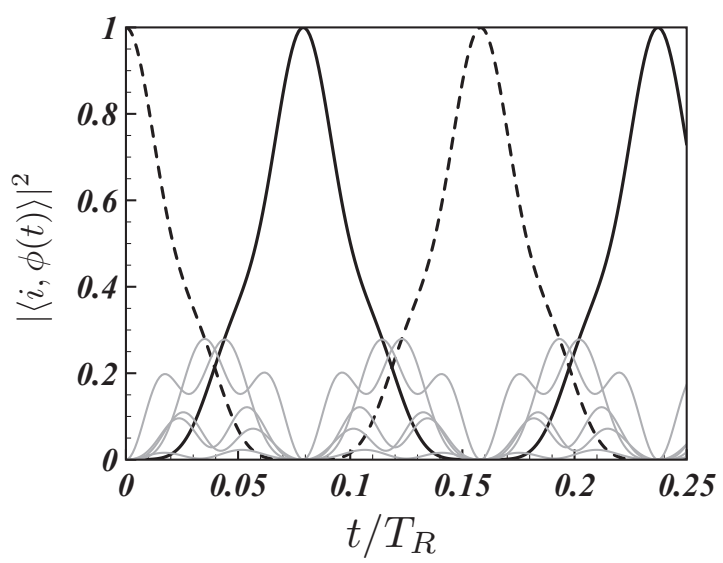

FIG. 1. Population dynamics of a near-to-optimal network conformation of coupled dipoles, from [46]. Mainly input (dashed black) and output (solid black) sites are populated during the dynamics, contrary to the bulk sites (gray), which exhibit weak populations never larger than approximately $30 \%$. Exactly this feature lies at the fundament of the dominant doublet design principle (see text).

Equation (7) is our final result for the transfer efficiency when only assuming centrosymmetry. Since eigenvectors $\left|\eta_{i}^{ \pm}\right\rangle$and eigenvalues $E_{i}^{ \pm}$are stochastic variables described by random matrix statistics, $\mathcal{P}_{H}$ will typically exhibit strong interference effects. While centrosymmetry tends to enhance the transfer efficiency via a mechanism related to weak localization [43], it still does not prevent the excitation to spread essentially uniformly over the network, as can be seen from the time averaged output site population:

$$
p_{H}=\lim _{T \rightarrow \infty} \frac{1}{T} \int_{0}^{T} d t \mid\left.\langle\text { out }, \phi(t)\rangle\right|^{2}=\sum_{i=1}^{N} \mid\left.\left\langle\text { out }, \eta_{i}\right\rangle\left\langle\eta_{i}, \text { in }\right\rangle\right|^{2} .
$$

Due to centrosymmetry, this can be rewritten as $p_{H}=$ $\sum_{i=1}^{N} \mid\left.\left\langle\right.$ in, $\left.\eta_{i}\right\rangle\right|^{4}$, a quantity closely related to the participation ratio [44]. From Ref. [45], one obtains for its ensemble average

$$
\overline{p_{H}}=\frac{3}{2+N} \text {. }
$$

This implies that, on average, at least $N / 3$ eigenvectors (with their associated eigenvalues) contribute to $\mathcal{P}_{H}$. While $\overline{p_{H}}$ and $\mathcal{P}_{H}$ are not trivially connected, it follows from (7) and (9) that optimal $\mathcal{P}_{H}$ can be accomplished only for optimal tuning of all these contributions, which is not guaranteed by centrosymmetry for individual realizations.

\section{B. Dominant doublet}

We therefore need to identify an additional design principle which turns an enhanced probability of efficient transport, as provided by centrosymmetry, into an almost certain event. Inspection of the structures of optimal Hamiltonians generated by a genetic algorithm [46] does not provide any obvious hint, but so does the time evolution of the populations of |in $\rangle$ and $\mid$ out $\rangle$, and of the bulk sites (see Fig. 1) which these Hamiltonians generate: Those of $\mid$ in $\rangle$ and |out $\rangle$ are strongly indicative of the tunneling dynamics in an effective double well potential, while the bulk sites exhibit comparably small, 
yet nonvanishing populations, with the same characteristic symmetry on the time axis.

This observation implies that random graphs with optimal transport properties exhibit a spectral property which we have labeled dominant doublet [18]: $|+\rangle$ and $|-\rangle$, as in (5), need to be close, in a sense to be quantified a bit further down, to eigenvectors $|\tilde{+}\rangle$ and $|\tilde{-}\rangle$ of $H^{+}$and $H^{-}$, respectively. Under this condition, the Hamiltonian (4) acquires the following, additional substructure:

$$
H=\left(\begin{array}{lccc}
E+V & \left\langle\mathcal{V}^{+}\right| & & \\
\left|\mathcal{V}^{+}\right\rangle & H_{\text {sub }}^{+} & & \\
& & E-V & \left\langle\mathcal{V}^{-}\right| \\
& & \left|\mathcal{V}^{-}\right\rangle & H_{\text {sub }}^{-}
\end{array}\right),
$$

with $\langle \pm|H| \pm\rangle=E \pm V$, and $\left|\mathcal{V}^{ \pm}\right\rangle$the couplings of the states $| \pm\rangle$ to the remainder of the system. The dominant doublet assumption further implies that $\left\|\mathcal{V}^{ \pm}\right\|$be sufficiently small.

Let us now exploit the dominant doublet property for a further simplification of (7). The dominant doublet's characteristic property being its dominant weight in the local density of states of the initial condition, i.e.,

$$
|\langle\underline{\underline{I}}, \pm\rangle|^{2}>\alpha \approx 1,
$$

implies that each of the two sums in (7) is dominated by a single term, thus

$$
\begin{aligned}
\mathcal{P}_{H} & \left.\approx \max _{t \in\left[0, T_{R}\right)} \frac{1}{4}\left|e^{-i t E^{+}}\right|\langle\tilde{+},+\rangle\right|^{2}-\left.e^{-i t E^{-}}|\langle\tilde{-},-\rangle|^{2}\right|^{2} \\
& \gtrsim \max _{t \in\left[0 . T_{R}\right)} \frac{2 \alpha-1}{4}\left|e^{-i t E^{+}}-e^{-i t E^{-}}\right|^{2}
\end{aligned}
$$

where $E^{ \pm}$in (12) is the eigenvalue associated with $|\tilde{ \pm}\rangle$. The energy difference $\left|E^{+}-E^{-}\right|$of the dominant doublet states, which is reduced or enhanced with respect to the direct coupling $V$ by the collective impact of the bulk sites, now acts as an effective tunneling rate that couples $\mid$ in $\rangle$ and $\mid$ out $\rangle$. At

$$
t_{0}=\frac{\pi}{\mid E^{+}-E^{-1}},
$$

the transfer probability is bounded from below by $2 \alpha-1$, and therefore large, since $\alpha \approx 1$. If, on top, $t_{0}<T_{R}$, then the excitation transfer is efficient in the sense defined above. We therefore need a quantitative prediction for $\left|E^{+}-E^{-}\right|$.

Under the dominant doublet assumption perturbation theory is a valid tool to study the problem. Perturbative techniques teach us that

$$
1-|\langle\tilde{ \pm}, \pm\rangle|^{2} \approx \sum_{i=1}^{N / 2-1} \frac{\left|\left\langle\mathcal{V}^{ \pm}, \psi_{i}^{ \pm}\right\rangle\right|^{2}}{\left(E \pm V-e_{i}^{ \pm}\right)^{2}},
$$

with $\left|\psi_{i}^{ \pm}\right\rangle$and $e_{i}^{ \pm}$the eigenvectors and eigenvalues of $H_{\mathrm{sub}}^{ \pm}$, respectively. Therefore, the requirement (11) implies a relation between $\alpha,\left|\left\langle\mathcal{V}^{ \pm}, \psi_{i}^{ \pm}\right\rangle\right|^{2}$, and $\left(E \pm V-e_{i}^{ \pm}\right)^{2}$. Furthermore, $E \pm V$ each is an eigenvalue up to an energy shift $s^{ \pm}$. This latter quantity can be obtained from standard perturbation theory, as

$$
s^{ \pm}=\sum_{i} \frac{\left|\left\langle\mathcal{V}^{ \pm}, \psi_{i}^{ \pm}\right\rangle\right|^{2}}{E \pm V-e_{i}^{ \pm}}, \text {such that } E^{ \pm}=E \pm V+s^{ \pm} .
$$

Notice that, for simplicity, we here present the expression that is obtained from nondegenerate perturbation theory. In the regime where $\left(E \pm V-e_{i}^{ \pm}\right) \approx 0$, we will need to consider a more complicated expression [see (30) in Sec. III C].

With $\Delta s=s^{+}-s^{-}$, it is clear that the effective tunneling rate $\left|E^{+}-E^{-}\right|$between $\mid$in $\rangle$and $\mid$out $\rangle$can be written as

$$
\left|E^{+}-E^{-}\right|=|2 V+\Delta s|,
$$

where the direct (Rabi-like) coupling term is now "renormalized" by the shift $\Delta s$ imparted by the cumulative effect of the randomly placed bulk sites of the graph. Large fluctuations thereof will induce large fluctuations of the transfer efficiency. Since the statistics of $\Delta s$ is inherited from the statistics of $H^{ \pm}$, we will be able to infer the statistics of the transfer efficiency, in the next chapter.

Before doing so, let us briefly comment qualitatively on which is the implication of the dominant doublet assumption for the excitation dynamics on the random graph: Imposing this mechanism, we greatly limited the freedom of the excitation to spread over the network, which quantum mechanically causes the typical delocalization over the different network sites as discussed at the end of the previous subsection. As apparent from a comparison of the spectral decompositions (7) and (12), the eigenvectors of the Hamiltonian tell the excitation where it is allowed to go, and the dominant doublet imposes a strong incentive for the excitation to go directly from input to output (or the other way round). Yet the time scale of the transport is set by the associated doublet eigenvalues, and these may be strongly affected by the remainder of the spectrum, via (15), as we will see hereafter.

\section{STATISTICS OF TRANSFER TIME SCALES}

We have so far reformulated our initial transport problem in terms of a spectral doublet structure which is amended by the perturbative coupling to some bulk states described by random matrices. This is a general scenario which is well known under the name chaos assisted tunnelling (CAT) [47] in the area of quantum chaos [5] and also reminiscent of transport problems in mesoscopic physics [3]. The fundamental idea is that the dynamical and/or transport properties in some predefined degree of freedom can be dramatically modified by the nonlinear coupling to some other degrees of freedom, incarnated, e.g., by a classical driving field [48-51], or by further coordinates of configuration space $[47,52,53]$. In the specific context of photosynthetic light harvesting, ideal candidates for such additional degrees of freedom are provided by those of the protein scaffold, which fix the boundary conditions for the electronic dynamics and excitations [17,26,54]. If these additional degrees of freedom themselves exhibit sufficiently complex dynamics, their coupling to the transporting degree of freedom will induce strong fluctuations in the transport properties of interest. We now import the random matrix theory (RMT) of CAT to derive analytical predictions for the statistics of the transfer efficiencies (12) and times (13), and in particular discuss the necessary amendments of the available theory to match the details of our model. 


\section{A. How to obtain the distribution of transfer times}

The distribution of $s^{ \pm}$is already known in terms of CAT, with $E, V=0$, and we will therefore strongly rely on the results of Refs. [51,55]. Note, however, that Ref. [56] has already argued under very general assumptions that the distribution of this type of quantity should always be a Cauchy distribution, irrespective of whether the $e_{i}^{ \pm}$strictly derive from GOE or from some other type of random Hamiltonian. This is important in our present context, since the biological functional units which inspire the present study are unlikely to realize GOE statistics in the strict sense. Moreover, Refs. [51,55] provide us with clear insight in the parameters determining the Cauchy distribution, for a setup which is close to ours. Adopting the mathematical language of Refs. [51,55], we obtain that, when $E=V=0$, the distribution of $s^{ \pm}$is given by

$$
\begin{aligned}
P\left(s^{ \pm}\right) & =\frac{1}{\pi} \frac{\sigma^{ \pm}}{\left(\sigma^{ \pm}\right)^{2}+\left(s^{ \pm}-s_{0}^{ \pm}\right)^{2}}=\operatorname{Cauchy}\left(s_{0}^{ \pm}, \sigma^{ \pm}\right), \\
\text {with } \sigma^{ \pm} & =\pi \frac{\left|\left\langle\mathcal{V}^{ \pm}, \psi_{i}^{ \pm}\right\rangle\right|^{2}}{\Delta}, \quad s_{0}^{ \pm}=0,
\end{aligned}
$$

where we assume that $\overline{\left|\left\langle\mathcal{V}^{ \pm}, \psi_{i}^{ \pm}\right\rangle\right|^{2}}=\overline{\left\|\mathcal{V}^{ \pm}\right\|^{2}}(N / 2-1)^{-1 / 2}$, with $\overline{\left\|\mathcal{V}^{ \pm}\right\|^{2}}$ a measure for the average interaction strength between $| \pm\rangle$ (and, thus, also $\mid$ in $\rangle$ and $\mid$ out $\rangle$ ) and the bulk states. The parameter $\Delta$ expresses the mean level spacing in the vicinity of $0[51,55]$.

In contrast to $E=V=0$ in Refs. [51,55], we need to accommodate for $E \pm V \neq 0$. This can be accomplished using the results of Ref. [57] and realizing that the curvatures presented in Eq. (5) of Ref. [57] are closely related to the energy shifts. Indeed, the shifts' distribution is given by Eq. (49) of Ref. [57], with $\sigma_{2}^{2}, \sigma^{2}$ and $\lambda$ in Ref. [57] substituted by $\overline{\left\|\mathcal{V}^{ \pm}\right\|^{2}}, 2 \xi^{2}$, and $E \pm V$, respectively, in our present nomenclature.

Note that, in Ref. [57], $\rho(\lambda)$ is the density of states, given by Wigner's semicircle [41] in the GOE. In the standard GOE scenario, the mean level spacing is known to change only slightly throughout the bulk of the spectrum, and it can be estimated by the radius of the semicircle [41]. In our present context, this would imply that we can use $\xi$ as a parameter just as well as $\Delta$. Note, however, that the matrices $H_{\text {sub }}^{ \pm}$ of our model are in general not GOE matrices, since they are obtained by postselection of that matrix subensemble of structure (4) which exhibits a dominant doublet as defined by (11). This means that the postselected ensemble does not obey Wigner-Dyson statistics, and thus $\rho(\lambda)$ is typically not the semicircle distribution. As essential consequence, the relation between the radius of the semicircle and the local mean level spacing no longer holds; we can no longer relate the global quantity $\xi$ to the local parameter $\Delta$ ! Moreover, it turns out, as extensively discussed in Sec. III C below, that $\Delta$ can vary strongly throughout the spectrum. In our derivation, the relevant quantity is the mean level spacing in the vicinity of $E \pm V$, which we will refer to as $\Delta_{\text {loc }}$.

Given (13) and (16), we need to infer the distribution of $\Delta s=s^{+}-s^{-}$. To do so, we can use simple properties of the Cauchy distribution. The fact that $s^{+} \sim \operatorname{Cauchy}\left(s_{0}^{+}, \sigma^{+}\right)$and $s^{-} \sim \operatorname{Cauchy}\left(s_{0}^{-}, \sigma^{-}\right)$implies that $s^{+}-s^{-} \sim \operatorname{Cauchy}\left(s_{0}^{+}-\right.$ $\left.s_{0}^{-}, \sigma^{+}+\sigma^{-}\right)$, which follows from the Cauchy distribution being a stable distribution [58]. In order to simplify notation, we define $s_{0}=s_{0}^{+}-s_{0}^{-}$and $\sigma=\sigma^{+}+\sigma^{-}$, to obtain

$$
\begin{aligned}
P(\Delta s) & =\frac{1}{\pi} \frac{\sigma}{\sigma^{2}+\left(\Delta s-s_{0}\right)^{2}}, \\
\text { with } \quad s_{0} & =2 V \frac{\overline{\left\|\mathcal{V}^{ \pm}\right\|^{2}}}{2 \xi^{2}}, \\
\text { and } \quad \sigma & =2 \pi \frac{\overline{\left\|\mathcal{V}^{ \pm}\right\|^{2}}}{(N / 2-1) \Delta_{\mathrm{loc}}},
\end{aligned}
$$

where we used that $\overline{\left\|\mathcal{V}^{+}\right\|^{2}}=\overline{\left\|\mathcal{V}^{-}\right\|^{2}}=\overline{\|\mathcal{V}\|^{2}}$. This follows from $\left\|\mathcal{V}^{+}\right\|^{2}$ and $\left\|\mathcal{V}^{-}\right\|^{2}$ being independent stochastic variables which are identically distributed, a property which they inherit from $\mathrm{H}^{+}$and $\mathrm{H}^{-}$being independent and identically distributed, and hence have the same expectation value.

The distribution of $\Delta s$ is but a first step to derive the distribution of $T_{R} / t$. The expressions for $t_{0}$ and $T_{R}$, using (13) and (16), imply that

$$
\frac{T_{R}}{t}=\left|1-\frac{\Delta s}{2 V}\right| .
$$

Since $E$ and $V$ are still considered to be fixed, we again use that the Cauchy distribution is stable [58]: This implies that, if $\Delta s \sim \operatorname{Cauchy}\left(s_{0}, \sigma\right)$, then

$$
1-\frac{\Delta s}{2 V} \sim \text { Cauchy }\left(1-\frac{s_{0}}{2 V}, \frac{\sigma}{2 V}\right) .
$$

The distribution of the absolute value $\left|1-\frac{\Delta s}{2 V}\right|$ thus reads

$$
\begin{aligned}
P\left(\left|1-\frac{\Delta s}{2 V}\right|=x\right)= & \frac{1}{\pi}\left[\frac{\gamma}{\gamma^{2}+\left(1+x_{0}+x\right)^{2}}\right. \\
& \left.+\frac{\gamma}{\gamma^{2}+\left(1+x_{0}-x\right)^{2}}\right], \\
\text { with } \quad x_{0}= & \frac{\left\|\mathcal{V}^{ \pm}\right\|^{2}}{2 \xi^{2}}, \\
\text { and } \quad \gamma= & \frac{1}{V} \frac{\pi \overline{\|\mathcal{V}\|^{2}}}{(N / 2-1) \Delta_{\text {loc }}} .
\end{aligned}
$$

We finally need to account for the fact that $E$ and $V$ are themselves stochastic variables, and we therefore need to average over their respective distributions. However, as shown in Sec. III D below, the probability distribution of $V$ is strongly peaked and, therefore, dominated by its mean value $\bar{V}$. Given this dominant behavior of the mean, it is usually a reasonable approximation to replace $V$ by $\bar{V}$ rather than exactly performing the integration. This approximation is what is called an annealed approximation [59] and leads to

$$
\gamma \approx \frac{1}{\bar{V}} \frac{\overline{\|\mathcal{V}\|^{2}}}{(N / 2-1) \Delta_{\text {loc }}},
$$

where $\Delta_{\text {loc }}$, the local mean level-spacing of energy levels in the vicinity of the energy $E \pm V$, and the value $\bar{V}$ still are to be determined.

Since the dominant doublet constraint modifies the local properties of the $H_{\text {sub }}^{ \pm}$ensemble around $E \pm V$, we cannot 
simply import the results available for GOE. We will therefore present a derivation of $\Delta_{\text {loc }}$ in Sec. III C hereafter and already warn the reader that this section will be rather technical and not extremely elegant, however, with the useful result

$$
\Delta_{\mathrm{loc}} \approx \frac{2 \pi \xi}{\sqrt{N / 2-1}} .
$$

Section III D below will provide the derivation of the parameter $\bar{V}$, which is mainly based on a Laplace approximation for the integration, and yields

$$
\bar{V} \approx \frac{2 \pi \xi}{e N \sqrt{N / 2-1}} .
$$

With the explicit expressions (23) and (24) in (22), we ultimately obtain from (21)

$$
\begin{aligned}
P\left(\frac{T_{R}}{t}=x\right)= & \frac{1}{\pi}\left[\frac{s_{0}}{s_{0}^{2}+\left(1+x_{0}+x\right)^{2}}\right. \\
& \left.+\frac{s_{0}}{s_{0}^{2}+\left(1+x_{0}-x\right)^{2}}\right], \\
\text { with } s_{0}= & \frac{\overline{\|\mathcal{V}\|^{2}} N e}{4 \pi \xi^{2}}, \\
\text { and } x_{0}= & \frac{\frac{\|\mathcal{V}\|^{2}}{2 \xi^{2}}}{}
\end{aligned}
$$

This is our final result for the distribution of the excitation transfer times generated by centrosymmetric Hamiltonians of the form (10) with dominant doublet strength $\alpha$. The relationship between $\alpha$, which is not explicit in (25), and $\overline{\|\mathcal{V}\|^{2}}$ will be derived in Sec. III C below; see (38).

\section{B. Scaling properties of characteristic transfer times}

From the thus obtained Cauchy distribution for $T_{R} / t$ we can obtain a good understanding of the probability of finding $\mathcal{P}_{H}$ close to one. According to (12), it is clear that $\mathcal{P}_{H} \geqslant 2 \alpha-1$ close to one if $t=\pi /|2 V+\Delta s|<T_{R}$. Therefore, we can infer the probability that $T_{R} / t$ is larger than one by straightforward integration over the corresponding range in (25). The result reads

$$
P\left(\frac{T_{R}}{t}>1\right)=1-\frac{1}{\pi} \arctan \left[\frac{4 \pi \xi^{2}}{\overline{\|\mathcal{V}\|^{2}} N e}\left(1-\frac{\overline{\|\mathcal{V}\|^{2}}}{2 \xi^{2}}\right)\right] .
$$

It follows that the probability for fast and efficient transport increases with the size $N$ of the network. As $N$ grows very large, we obtain

$$
P\left(\frac{T_{R}}{t}>1\right) \approx 1-\frac{4 \xi^{2}}{\overline{\|\mathcal{V}\|^{2}} N e} .
$$

In other words, the tail of the distribution in Eq. (25) grows heavier with increasing $N$ and therefore more and more realizations enhance the transport. The origin of this scaling can be traced back to the direct (in-out) coupling $V$, since $N$ enters through $\bar{V}$. The coupling is the smallest number in absolute value of a set of $N / 2$ normally distributed variables, and, as explained in Sec. IIID below, for a fixed density of states its expectation value decreases $\propto N^{-3 / 2}$, in leading order.
In large systems, the direct tunneling from input to output will be negligible, and the intermediate sites provide a considerable boost to the transport (much in the spirit of CAT [47]). Thus, if we compare the time scale of the direct coupling, $T_{R}$, to the effective transport time $t$, we should find $t<T_{R}$ with high probability. This intuition perfectly matches the result displayed in Fig. 5 below.

Alternatively, when studying systems where the direct coupling is fixed to a value $V^{*}$ for all realizations of the networks' conformation, a very different scaling is obtained [by suitable integration of Eq. (21), rather than of (25), due to the explicit dependence on $V$ in (21)]:

$$
\begin{aligned}
& P\left(\frac{T_{R}}{t}>1\right) \\
& \quad=1-\frac{1}{\pi} \arctan \left[\frac{2 V^{*} \xi \sqrt{N / 2-1}}{\overline{\|\mathcal{V}\|^{2}}}\left(1-\frac{\overline{\|\mathcal{V}\|^{2}}}{2 \xi^{2}}\right)\right] .
\end{aligned}
$$

Now we find that, in the limit of large $N$, this expression scales as

$$
P\left(\frac{T_{R}}{t}>1\right) \approx \frac{1}{2}+\frac{\overline{\|\mathcal{V}\|^{2}}}{\pi V^{*} \xi \sqrt{2 N}}
$$

i.e., the relative weight of conformations which enhance the transport decreases with $N$, though remains bounded from below by $50 \%$. Since, in this regime, the direct tunneling from |in $\rangle$ to $\mid$ out $\rangle$ always has the same strength, we can thus conclude that increasing the system size in this postselected ensemble has a negative impact on the chaos-assisted tunneling contribution to the transport; the peak around $T_{R} / t=1$ in the Cauchy distribution (21) is enhanced at the expense of the tail.

The two asymptotic scaling laws (27) and (29) can be given a more physical interpretation: If, as in the molecular networks at the heart of photosynthetic light-harvesting (which inspired our model), coupling strength is synonymous to spatial separation, then increasing $N$ at fixed spatial density, and thus literally increasing the spatial size of the network, leads to (27). Alternatively, keeping the spatial size of the network fixed and increasing the packing density by increasing $N$ leads to (29).

In closing this part of our discussion, let us also emphasize that the probability given in (26) is only a lower bound of the probability to obtain $\mathcal{P}_{H}>2 \alpha-1$. In order to understand this, let us reconsider Eq. (12): The time $t=\pi /|2 V+\Delta s|$ is the point in time when $\left|\exp \left(-i t E^{+}\right)-\exp \left(-i t E^{-}\right)\right|^{2} / 4$ reaches its largest possible value. Nevertheless, for a specific realization of the disorder, we may find other (and in particular earlier) moments in time at which already $\left|\exp \left(-i t E^{+}\right)-\exp \left(-i t E^{-}\right)\right|^{2} / 4>2 \alpha-1$. These realizations are not included in (26) [which was derived by using the relation (13) and (16)], although $\mathcal{P}_{H}>2 \alpha-1$. As an example, Fig. 2 shows a realization of the time dependence of the output population for which $T_{R} / t=0.970874$ and indicates the value $2 \alpha-1$ by a dashed line. Since $t>T_{R}$, we do not account for this in our estimate (26) of efficient realizations, even though it clearly exhibits $\mathcal{P}_{H}>2 \alpha-1$.

As a final remark in this section, and as an important intermediate result, let us emphasize that the desired transport properties of the network as described above do not depend on 


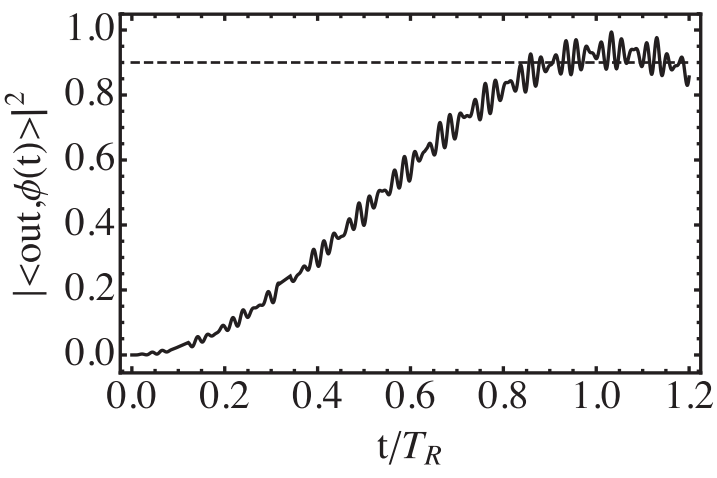

FIG. 2. Probability $\mid\left.\langle$ out,$\phi(t)\rangle\right|^{2}$ to find the excitation at the output site, for a single realization of the network Hamiltonian (10). The value $2 \alpha-1$ is indicated by a dashed line. Even though the transfer time $t=1.03 T_{R}$ for this realization, $\mathcal{P}_{H}>2 \alpha-1$.

the details of the individual networks' structures. Indeed, only course-grained and somewhat easily controllable quantities, the spectral density $\xi$ of the bulk states, and the average coupling strength $\overline{\|\mathcal{V}\|^{2}}$ of the input and output site to the bulk, fully determine the distribution (25).

\section{The mean level spacing $\Delta_{\text {loc }}$ in the vicinity of $E \pm V$}

Now that a global picture has been established, we need to understand the technical details required to obtain an expression for $\Delta_{\text {loc }}$, the mean level spacing near the energy $E \pm V$ which entered (25) through (21) and (22). It was already indicated in Sec. II that the dominant doublet constraint is somewhat more subtle than the mechanism of chaos assisted tunneling, where this mean level spacing is known a priori. The dominant doublet in our model can be seen as a strong demand of eigenvector localization (11). Since, in our present work, we sample centrosymmetric Hamiltonians and postselect realizations where a dominant doublet is present, a strong modification of the local mean level spacing around the energy $E \pm V$ can be induced. This effect is also apparent from the density of states, shown in Fig. 3: Wigner's semicircle, to be expected from RMT [41], is garnished by a cusp, centered around $E+V$ (in the figure fixed at $E+V=1)$. The key

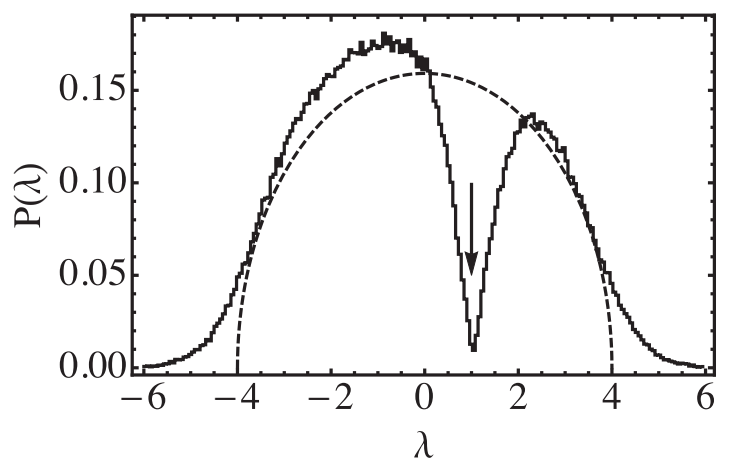

FIG. 3. Density of states of $H_{\text {sub }}^{+}$, for $N=10$ and $\xi=2$, with fixed $E+V=1$ (arrow) to highlight the effect of a dominant doublet in the vicinity of this energy level. In contrast to the Wigner semicircle (dashed line), valid for the GOE ensemble with $N \rightarrow \infty$, the density of states exhibits a cusp at $\lambda=E+V$. approach to deriving an estimate for $\Delta_{\text {loc }}$ is the assumption that it is essentially the same quantity as the width of the cusp, which we now set out to determine.

To reach a quantitative understanding of the cusp effect, we must be able to treat the eigenvalues $e_{i}^{ \pm}$of $H^{ \pm}$which are close to $E \pm V$. Here, we find strong repulsion between the energy levels, causing the cusp. For an exact description of the effect, we must include the possibility of (near-)degeneracy between $E \pm V$ and one of the $e_{i}^{ \pm}$and thus use degenerate perturbation theory. Therefore, we first consider the degenerate variant of (14):

$$
1-|\langle\underline{ \pm}, \pm\rangle|^{2} \approx \frac{1}{2} \sum_{i=1}^{N / 2-1}\left\{1-\left[1+4 \frac{\left|\left\langle\mathcal{V}^{ \pm}, \psi_{i}^{ \pm}\right\rangle\right|^{2}}{\left(E \pm V-e_{i}^{ \pm}\right)^{2}}\right]^{-1 / 2}\right\} .
$$

All special effects caused by the dominant doublet originate from this expression, via (11). The requirement that the lefthand side of (30) be smaller than $1-\alpha$ imposes constraints on the possible values which

$$
D:=\min _{i}\left|E \pm V-e_{i}^{ \pm}\right|
$$

can take. As the quantity $D$ is directly related to the cusp in Fig. 3, it will form the cornerstone to our estimate of $\Delta_{\text {loc }}$.

First, we observe that there are three parameters in (30) which must be controlled to fulfill the dominant doublet constraint: $D,\|\mathcal{V}\|^{2}$, and $\alpha$. Of these, only the last one is controlled directly in our setup. Looking at the right-hand side of (30), one sees that the dominant doublet regime is reached for $\|\mathcal{V}\| / D$ sufficiently small, such that this right-hand side of the equation vanishes and $1-|\langle\mathcal{I}, \pm\rangle|^{2}$ is close to zero. While $\|\mathcal{V}\|^{2}$ can be measured rather straightforwardly in our simulations, we require an estimate of $D$ in terms of the other known parameters: $\alpha, \xi$, and $\overline{\|\mathcal{V}\|^{2}}$.

To obtain such an estimate, we focus on two limiting cases, which we both expect to encounter in the same ensemble of postselected Hamiltonians. Moreover, each of these cases will impose constraints on the possible range of the parameters $\|\mathcal{V}\|$ and $D$. As mentioned, the dominant doublet implies that $\|\mathcal{V}\| / D$ should be small, which implies that $\|\mathcal{V}\|$ is sufficiently small, or that $D$ is sufficiently large. The two limiting cases exactly boil down to these scenarios: In the first limiting case, we will consider Hamiltonians where all eigenvalues $e_{i}^{ \pm}$are outside of the cusp region of Fig. 3. In this regime, the dominant doublet imposes constraints on $\|\mathcal{V}\|$. In the other limiting case, we investigate what happens when one of the $e_{i}^{ \pm}$lingers inside the cusp region of Fig. 3, which leads to constraints on $D$. Throughout these calculations, even though mathematically somewhat unsound, we assume that $\|\mathcal{V}\|$ and $D$ are two independent statistical quantities. Finally, once the two limiting scenarios have been considered, we combine the two constraints, as they should both hold for the complete ensemble, and formulate an estimate for the width of the cusp.

The first limiting case is given by network realizations where all eigenvalues $e_{i}^{ \pm}$exhibit a considerable distance from $E \pm V$, far from the observed cusp in Fig. 3. Therefore, all terms in the sum (30) contribute equally. This leads to the approximation that the expectation value of a single one of these terms is $(1-\alpha) /(N / 2-1)$. Rather than (30), we can 
then use (14), i.e.,

$$
1-|\langle\tilde{ \pm}, \pm\rangle|^{2} \approx \sum_{i=1}^{N / 2-1} \frac{\left|\left\langle\mathcal{V}^{ \pm}, \psi_{i}^{ \pm}\right\rangle\right|^{2}}{\left(E \pm V-e_{i}^{ \pm}\right)^{2}} .
$$

On the level of averages, the dominant doublet condition tells us thus that

$$
\overline{\frac{1-\alpha}{N / 2-1}} \approx \overline{\left[\frac{\left|\left\langle\mathcal{V}^{ \pm}, \psi_{i}^{ \pm}\right\rangle\right|^{2}}{\left(E \pm V-e_{i}^{ \pm}\right)^{2}}\right]} .
$$

Here we assume that, since the $e_{i}^{ \pm}$stay far away from the cusp, and therefore do not feel the "repulsion" from $E \pm V, E$ and $V$ can be approximately treated as independent variables. The variance of $V$, as its statistics is described by extreme value theory [60], see Sec. IIID below, is neglected as the distribution of $V$ is strongly peaked around $\bar{V}$. Furthermore, we approximate the distribution of the $e_{i}$ (locally) by a semicircle law. The crude approximation that each term in (14) provides a similar contribution leads to

$$
\sqrt{\frac{1-|\langle \pm, \pm\rangle|^{2}}{N / 2-1}} \approx \frac{\left|\left\langle\mathcal{V}^{ \pm}, \psi_{i}^{ \pm}\right\rangle\right|}{\left|E \pm V-e_{i}^{ \pm}\right|}, \text {for all } i .
$$

Comparing (33) to (34), we get $\overline{1-|\langle\Psi, \pm\rangle|^{2}} \approx 1-\alpha$. If we now assume that $\left\langle\mathcal{V}^{ \pm}, \psi_{i}^{ \pm}\right\rangle$is normally distributed, with zero mean and variance $\|\mathcal{V}\|^{2} /(N / 2-1)$, and that the $e_{i}$ obey a semicircle law, as one expects from RMT [55], we find

$$
\sqrt{1-\alpha} \approx \sqrt{\frac{2 \| \overline{\mathcal{V} \|^{2}}}{\pi \xi^{2}}} .
$$

We validate this result by numerical data (see Sec. IV) and approximate

$$
\alpha \approx 1-C \frac{\overline{\|\mathcal{V}\|^{2}}}{\xi^{2}}
$$

with $C$ as a fit parameter. The numerical dataset is obtained by scanning $\alpha$ from 0.99 to 0.8 , for fixed $\xi=2$ and $N=14$.

For each value of $\alpha$ we extract $\overline{\|\mathcal{V}\|^{2}}$. We also inspected data with $\alpha \in[0.94,0.99], \xi=20$, and $N=10$. Figure 4 suggests a linear dependence as in (36). However, since the ansatz (36)

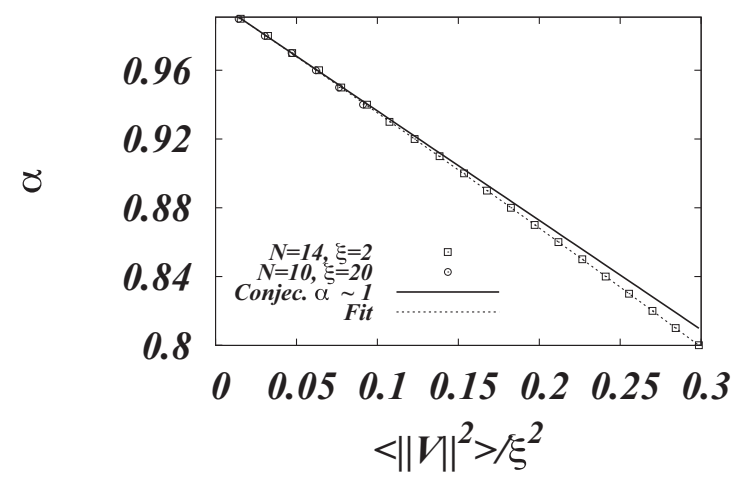

FIG. 4. Dependence of $\alpha$ on $\overline{\|\mathcal{V}\|^{2}} / \xi^{2}$, for different network realizations. In order to extract the constant $C$ in (36) and (37), a fit is performed. The conjectured curve for $\alpha \approx 1$, where $C=2 / \pi$, is given by the solid line. results from perturbation theory, it appears reasonable to add a term quadratic in $\overline{\|\mathcal{V}\|^{2}} / \xi^{2}$, for $\alpha \approx 0.8$. We thus fit the data to the form

$$
\alpha \approx 1-C \frac{\overline{\|\mathcal{V}\|^{2}}}{\xi^{2}}-b\left(\frac{\overline{\|\mathcal{V}\|^{2}}}{\xi^{2}}\right)^{2}
$$

and obtain the following result:

\begin{tabular}{l|ll} 
& Estimate & Standard Error \\
\hline$C$ & 0.636789 & 0.00218418 \\
$b$ & 0.111501 & 0.00933118
\end{tabular}

By definition (11), the dominant doublet is found where $\alpha \approx 1$ and thus $\overline{\|\mathcal{V}\|^{2}} / \xi^{2}$ is small. Therefore we can finally ignore the second order term in Eq. (37) and obtain that $\alpha \approx 1-$ $0.636789 \times \overline{\|\mathcal{V}\|^{2}} / \xi^{2}$. As the estimate $C \approx 2 / \pi$ falls into the error margin of our numerically generated data, we conclude that

$$
1-\alpha \approx \frac{2 \overline{\|\mathcal{V}\|^{2}}}{\pi \xi^{2}} .
$$

Since this limiting case was defined above as the one where the eigenvalues $e_{i}^{ \pm}$are far away from the cusp and thus do not experience the repulsion which must be felt as they approach $E \pm V$ (this exactly causes the cusp seen in Fig. 3), we will consider a second limiting scenario in order to probe the smallest possible value of $D$ as given by (31).

The second limiting case is when one eigenvalue $e_{i}^{ \pm}$ approaches $E \pm V$ at a minimum distance $D_{\min }$ (where the minimum is taken over the entire ensemble) such that we find the dominant doublet with probability one, with $\left\langle\psi_{i}^{ \pm}, \mathcal{V}^{ \pm}\right\rangle$still a normally distributed stochastic variable. This implies that the sum in (30) be dominated by a single term. Ultimately our goal is to determine $D_{\min }$, and to do so we study the statistics of a single term $\tau$ in (30), leaving the resonance denominator, (31), as a free parameter. This term is a stochastic quantity, and we can obtain its probability density as

$$
P_{D}(\tau)=\int_{\mathbb{R}} d v N(v) \delta\left\{\tau-\frac{1}{2}\left[1-\left(1+4 \frac{v^{2}}{D^{2}}\right)^{-1 / 2}\right]\right\} .
$$

$v=\left\langle\mathcal{V}^{ \pm}, \psi_{i}^{ \pm}\right\rangle$is again normally distributed, with zero mean and variance $\overline{\|\mathcal{V}\|^{2}} /(N / 2-1)$, and we denote the Gaussian probability density function by $N(v)$.

The integration can be performed straightforwardly using properties of the Dirac delta function. As the dominant doublet arises in a regime where $1-\alpha \approx 0$, we obtain from (30) that also $\tau$ must me close to zero; hence we can focus on the leading scaling behavior in $\tau \rightarrow 0$, from which we obtain

$$
P_{D}(\tau) \approx \frac{D \sqrt{N-2}}{4 \sqrt{\pi \overline{\|\mathcal{V}\|^{2}} \tau}} .
$$

Remember that the dominant doublet was imposed as a strict constraint (11), which implies that $|\langle\underline{ \pm}, \pm\rangle|^{2}$ is always larger than $\alpha$. Since we are now studying the case where one term $\tau$ dominates the sum in (30), $\tau$ must always be smaller than 
$1-\alpha$. This condition translates to

$$
\operatorname{Prob}(\tau \leqslant 1-\alpha)=\int_{0}^{(1-\alpha)} d t P_{D}(\tau)=1,
$$

and, with (40), defines an equation which can be solved to obtain the smallest possible value for $D$, which is denoted by $D_{\min }$ :

$$
D_{\min }=\frac{\sqrt{2 \pi \overline{\|\mathcal{V}\|^{2}}}}{\sqrt{(1-\alpha)(N / 2-1)}} .
$$

$D_{\text {min }}$ gives the closest allowed distance between $E \pm V$ and an eigenvalue $e_{i}$ of $H_{\text {sub }}^{ \pm}$to ensure $|\langle\tilde{ \pm}, \pm\rangle|^{2}>\alpha$.

Combining the constraints (35) and (42), which connect the parameters $\alpha, \overline{\|\mathcal{V}\|^{2}}, \xi$ and $D_{\min }$, we can express $D_{\min }$ as

$$
D_{\min }=\frac{\pi \xi}{\sqrt{N / 2-1}} .
$$

With (43), we obtain the following strong conjecture for $\Delta_{\text {loc }}$ :

$$
\Delta_{\mathrm{loc}} \approx \frac{2 \pi \xi}{\sqrt{N / 2-1}},
$$

where we used that $D_{\min }$ is the minimal distance between $E \pm V$ and an eigenvalue $e_{i}^{ \pm}$which can establish a dominant doublet. Since we are interested in the distance between two eigenvalues $e_{i}^{ \pm}$and $e_{j}^{ \pm}$, which we approximate by the width of the cusp in Fig. 3, we acquire an extra factor two, leading to $\Delta_{\text {loc }} \approx 2 D_{\text {min }}$ (much as in the elementary theory of level repulsion at degeneracy).

\section{The expectation value of the direct coupling}

The last parameter which remains to be estimated, is the expectation value of the direct in-out coupling, $\bar{V}$. Rather than obeying Gaussian statistics such as the coupling between any other two sites of the network, $V$ is governed by so-called extreme value statistics [60]. This is implicitly imposed by construction, since we defined $V=\min _{i}\left|H_{i, N-i+1}\right|$, which is the smallest number, in absolute value, of a sample of $N / 2$ normally distributed stochastic variables (the Hamiltonian components $H_{i, N-i+1}$ ). To calculate $\bar{V}$, we start by introducing a method to obtain the distribution of $V$, which we introduce in a general framework and subsequently apply to our specific problem.

To begin with, let $X_{1}, \ldots, X_{n}$ be a sample of $n$ independent, identically distributed stochastic variables, and denote $m=$ $\min _{k \in\{1, \ldots n\}} X_{k}$. We are now interested in the probability density $P_{m}(x)=P(m=x)$. To obtain this function, we consider the cumulative distribution function (CDF) of $m$, $F_{m}(x)=P(m \leqslant x)$. Since $m$ is the minimum

$$
\begin{aligned}
F_{m}(x)=P(m \leqslant x) & =1-\prod_{k=1}^{n} P\left(X_{k}>x\right) \\
& =1-\prod_{k=1}^{n}\left[1-P\left(X_{k} \leqslant x\right)\right] \\
& =1-(1-F(x))^{n},
\end{aligned}
$$

where $F(x)$ is the CDF of $X_{k}$. Now the probability density $P_{m}(x)$ can be obtained as

$$
P_{m}(x)=\frac{d F_{m}(x)}{d x}=1-\frac{d}{d x}[1-F(x)]^{n},
$$

which is seen to strongly depend on the sample size $n$.

In the present case we are dealing with $X_{k}=\left|H_{k, N-k+1}\right|$ and $H_{k, N-k+1} \sim \mathcal{N}\left(0, \frac{2 \xi^{2}}{N}\right)$ [recall (1)], what implies that $\left|H_{k, N-k+1}\right|$ is a half-normal distribution [61], therefore the $\mathrm{CDF}$ is given by

$$
\begin{aligned}
F_{\left|H_{k, N-k+1}\right|}(x) & =\frac{2}{\sqrt{\pi}} \int_{0}^{x} d\left|H_{k, N-k+1}\right| e^{-\left(\frac{\sqrt{N\left|H_{i, N-i+1}\right|}}{2 \xi}\right)^{2}} \\
& =\operatorname{erf}\left(\frac{\sqrt{N} x}{2 \xi}\right)
\end{aligned}
$$

where $\operatorname{erf}(x)$ denotes the error function [62]. By using this result and $n=\frac{N}{2}$ in (44) and (45), we obtain that the probability density of the minimal coupling $V$ is given by

$$
P(V)=\frac{e^{-\frac{N V^{2}}{4 \xi^{2}}} N^{3 / 2}\left[\operatorname{erfc}\left(\frac{\sqrt{N} V}{2 \xi}\right)\right]^{\frac{N}{2}-1}}{2 \sqrt{\pi} \xi},
$$

with erfc(x) the complementary error function, which is given by $\operatorname{erfc}(\mathrm{x})=1-\operatorname{erf}(\mathrm{x})[62]$.

From these results, $\bar{V}$ is now inferred as

$$
\begin{aligned}
\bar{V} & =\int_{0}^{\infty} d V P(V) V \\
& =\int_{0}^{\infty} d V \frac{e^{-\frac{N V^{2}}{4 \xi^{2}}} N^{3 / 2}\left[\operatorname{erfc}\left(\frac{\sqrt{N} V}{2 \xi}\right)\right]^{\frac{N}{2}-1}}{2 \sqrt{\pi} \xi} V .
\end{aligned}
$$

With the change of variable

$$
V^{\prime}=\frac{\sqrt{N} V}{2 \xi},
$$

the right-hand side of (48) turns into

$$
\frac{2 \xi \sqrt{N}}{\sqrt{\pi}} \int_{0}^{\infty} d V^{\prime} e^{-V^{\prime 2}}\left[\operatorname{erfc}\left(V^{\prime}\right)\right]^{\frac{N}{2}-1} V^{\prime} .
$$

Since we are interested in the behavior for large $N$, we have $N / 2-1 \approx N / 2$. We now apply Laplace's method [63] and thus need to define a function $f$ such that

$$
\int_{0}^{\infty} d V^{\prime} e^{-V^{\prime 2}}\left[\operatorname{erfc}\left(V^{\prime}\right)\right]^{\frac{N}{2}-1} V^{\prime}=\int_{0}^{\infty} d V^{\prime} \exp \left[N f\left(V^{\prime}\right)\right]
$$

It is straightforward to check that

$$
f\left(V^{\prime}\right)=-\frac{V^{\prime 2}}{N}+\left(\frac{1}{2}-\frac{1}{N}\right) \log \left[\operatorname{erfc}\left(V^{\prime}\right)\right]+\frac{1}{N} \log V^{\prime}
$$

is a suitable choice. In order to apply Laplace's method, we need to find that $V_{0}$ for which $f$ is extremal, hence $f^{\prime}\left(V_{0}\right)=0$. 
By straightforward calculation of the derivative of (52) we find

$$
f^{\prime}\left(V^{\prime}\right)=\frac{1}{N V^{\prime}}-\frac{2 V^{\prime}}{N}-\left(1-\frac{2}{N}\right) \frac{e^{-V^{\prime 2}}}{\sqrt{\pi} \operatorname{erfc}\left(V^{\prime}\right)},
$$

which allows only for an implicit expression for $V_{0}$. We can, however, get an explicit result by the following approximation: As the maximum of $f\left(V^{\prime}\right)$ is achieved for $V_{0} \ll 1$, we can expand $e^{-V^{\prime 2}}$ and $\operatorname{erfc}\left(\mathrm{V}^{\prime}\right)$ around $V^{\prime} \approx 0$, in order to obtain a tractable approximation for $f\left(V^{\prime}\right)$. This expansion yields

$$
\frac{e^{-V^{\prime 2}}}{\sqrt{\pi} \operatorname{erfc}\left(V^{\prime}\right)}=\frac{1-V^{\prime 2}+\frac{1}{2} V^{\prime 4}-\cdots}{\sqrt{\pi}\left(1-2 V^{\prime}+\frac{2}{3} V^{\prime 3}+\cdots\right)} \approx \frac{1}{\sqrt{\pi}} .
$$

Even though this is a rough approximation, the corrections due to higher orders are negligible for large $N$, numerical evaluation of (48) shows that, even for $N=10$, the exact results are very well approximated by (54).

With the low order approximation of $(54), f^{\prime}\left(V_{0}\right)=0$ is satisfied for

$$
V_{0} \approx \frac{\sqrt{N^{2}+8 \pi}-N}{4 \sqrt{\pi}} \approx \frac{\sqrt{\pi}}{N}\left(1+\frac{2}{N}\right),
$$

and Laplace's method now tells us that

$$
\int_{0}^{\infty} d V^{\prime} \exp \left[N f\left(V^{\prime}\right)\right] \approx \mathrm{e}^{N f\left(V_{0}\right)} \sqrt{\frac{2 \pi}{N\left|f^{\prime \prime}\left(V_{0}\right)\right|}},
$$

leading to the final result,

$$
\bar{V} \approx \frac{2 \pi \xi}{e N \sqrt{N / 2-1}}
$$
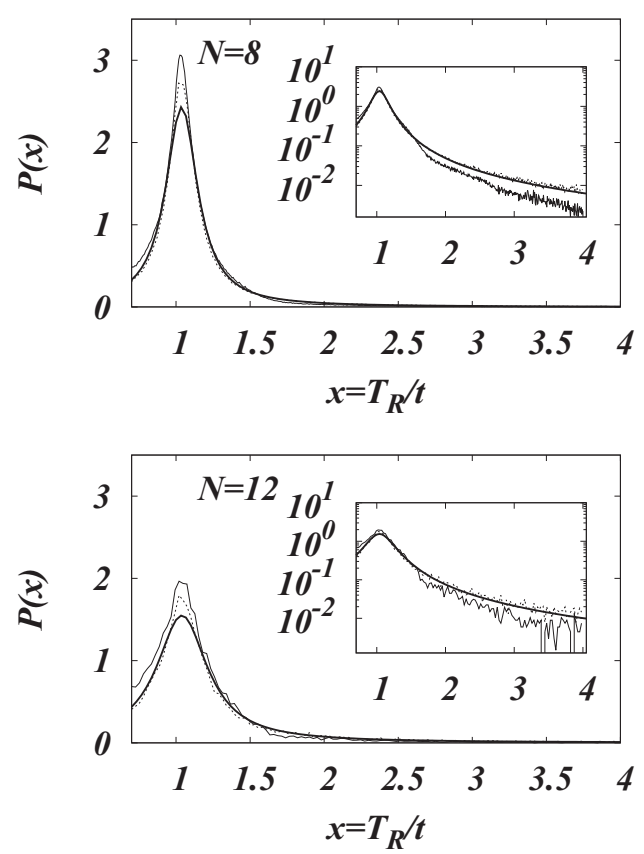

which we already anticipated in Sec. III A, to obtain the transfer time distribution (25).

\section{SIMULATIONS FOR RANDOM HAMILTONIANS}

Having completed the derivation of the analytical predictions of our constrained (by centrosymmetry and dominant doublet assumption) RMT model for efficient transport on random graphs, we now test these predictions against numerical simulations. We sample random Hamiltonians from the GOE, with centrosymmetry imposed as an extra constraint. After diagonalization of each of these Hamiltonians, we postselect those which exhibit a dominant doublet with weight $\alpha$, as defined in (11). Then, from the thus constructed RMTensemble, we numerically derive $\mathcal{P}_{H}$, with $t$ the earliest point in time for which $\mid\left.\langle$ out, $\phi(t)\rangle\right|^{2}=\mathcal{P}_{H}$.

To start, Fig. 5 shows the transfer time distribution for different network sizes $N$, at fixed spatial density [remember our discussion of (27) and (29) above], with a comparison between numerical data (thin solid line) and the analytical prediction (25) (thick solid line). There are no fitting parameters; the average coupling strength $\overline{\|\mathcal{V}\|^{2}}$ is directly extracted from the statistical sample, whereas the dominant doublet strength $\alpha=0.95$ and spectral density $\xi=2$ (in units of mean level spacing) are fixed a priori for all realizations.

The overall comparison of numerical data and analytical prediction is very satisfactory. In particular, the distribution also exhibits the trend predicted by (25) and (27) for increasing $N$ : As $N$ grows, the height of the maximum of the distribution at $T_{R} / t \approx 1$, controlled by $s_{0}$ [see (25)] decreases, and the
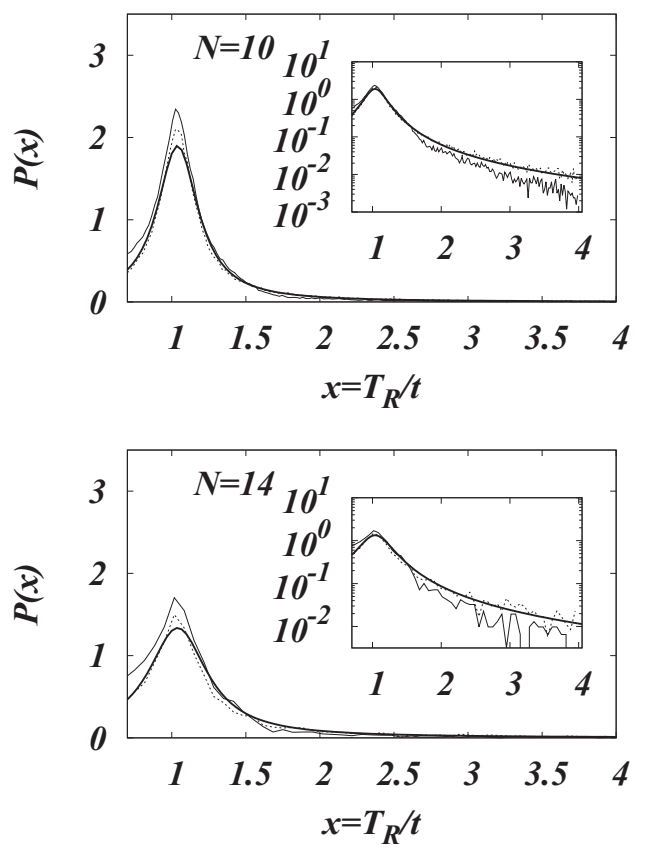

FIG. 5. Histograms of the simulated inverse transfer time $T_{R} / t$ (thin solid lines) across fully connected random networks of variable size $N$, and of $\left|E^{+}-E^{-}\right| / 2 V$ (dashed lines), together with the theoretical distribution (25) (thick solid line). The parameters $\xi=2$ and $\alpha=0.95$ are fixed for every realization. The value $\overline{\|\mathcal{V}\|^{2}} \approx 0.31$ is extracted from the simulations for each value of $N$. The simulations consider only a time window $\left[0,1.7 T_{R}\right]$, therefore the minimum value of the inverse transfer time is given by $T_{R} / t=(1.7)^{-1}$. The inset stresses the agreement between the theoretically predicted algebraic tail (thick solid line) and the $\left|E^{+}-E^{-}\right| / 2 V$ histogram (dashed line). The histogram for $T_{R} / t$ (thin solid line) slightly deviates from the other two curves because the quasiperiodicity of the dynamics suppresses the tail of the distribution (see text). 


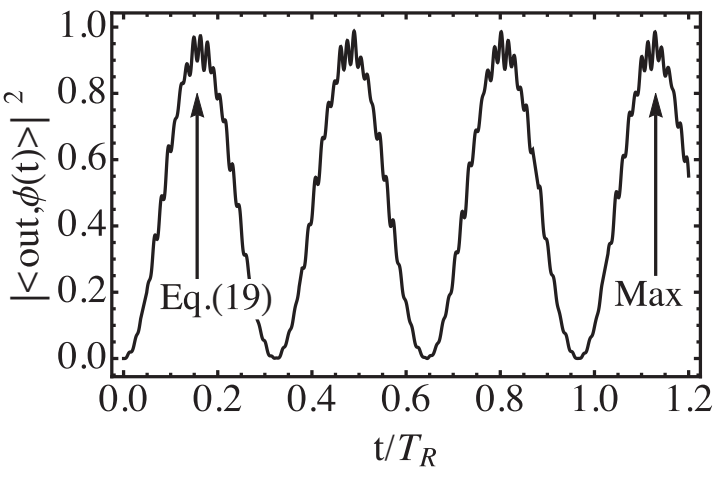

FIG. 6. Probability $\mid\left.\langle$ out, $\phi(t)\rangle\right|^{2}$ to find the excitation at the output site, for a single realization of the Hamiltonian (10). There are multiple strong localizations at the output site within $\left[0, T_{R}\right)$. High-frequency oscillations show that the dynamics is quasiperiodic rather than periodic.

algebraic tail with $T_{R} / t \gg 1$ grows fatter, as anticipated by (27). Indeed, the numerical data confirm the predicted scaling of $s_{0}$ and $P\left(T_{R} / t>1\right)$ with $N^{-1}$, as spelled out by Fig. 8.

However, closer scrutiny of the displayed distributions for larger values of $T_{R} / t$ (see the insets of Fig. 5) suggests an apparent discrepancy between numerics and analytical prediction: The numerical data appear to drop faster with increasing $T_{R} / t$ than expected from (25), which was derived from the statistics of the first passage time (13). It turns out that this is an effect caused by the quasiperiodic oscillation between the input and the output site. If, e.g., $T_{R} / t>3$, the excitation will localize on the output site three times during the benchmark time interval $\left[0, T_{R}\right)$. Since, however, the dynamics
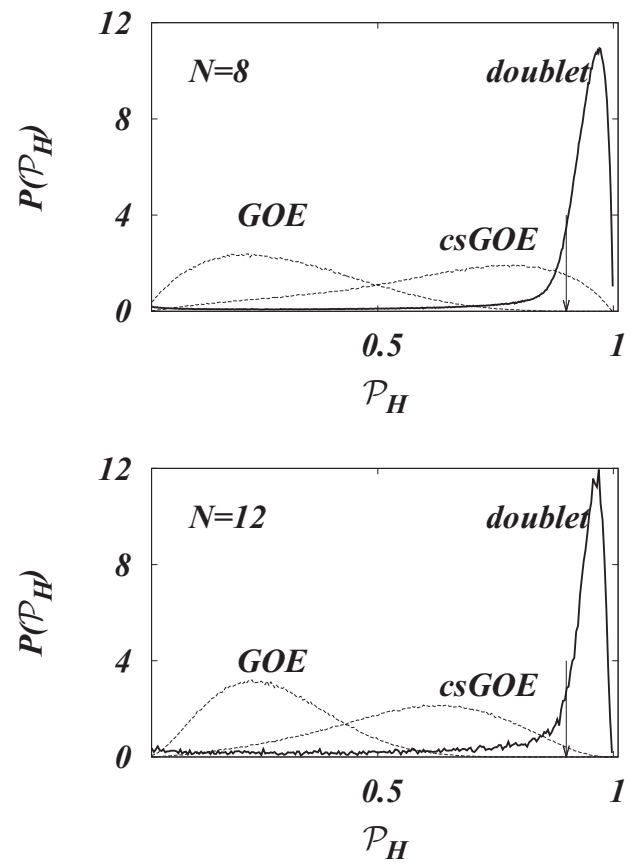

is in general quasiperiodic, rather than periodic (note that this is a consequence of the transient population of the bulk sites, which is neglected in the approximate expression (13) for the transfer time in terms of the dominant doublet splitting), the largest value of $\mid\left.\langle$ out,$\phi(t)\rangle\right|^{2}$ within the considered time window may only be achieved after multiple periods. Even though the theoretical value of $t$ is relatively small, the simulation may pick up a later point in time, thus giving a smaller weight to large values of $T_{R} / t$ in the histograms of Fig. 5. One incident of this scenario is shown in Fig. 6.

Indeed, direct comparison of the time scale (13) given by the numerically sampled doublet splitting (16) (rather than of $t$ as inferred from direct propagation of the associated unitary generated by $H$ ) leads to perfect agreement in particular of the asymptotic behavior of the distribution with the analytical prediction, as evident from comparison of the dotted and full curves in Fig. 5. The dominant doublet mechanism is thus impressively confirmed, with an asymptotic behavior inherited from the statistics of the level shifts $\Delta s$, induced by the interaction with the network's bulk sites.

Having achieved an excellent understanding of the transfer time distribution of centrosymmetric random graphs with dominant doublet, we still need to verify that they indeed also generate large transfer probabilities $\mathcal{P}_{H} \geqslant 2 \alpha-1 \approx 0.9$, for the chosen dominant doublet strength $\alpha=0.95$. This is done in Fig. 7, for the same model parameters as in Fig. 5, and in comparison to the efficiency distribution of unconstrained or just centrosymmetric random graphs (obeying GOE statistics). For different values of $N$, the figure provides an impressive illustration of the here suggested design principles: centrosymmetry alone already induces a very tangible shift of the average value of the transfer efficiency to much enhanced values though fails to concentrate the distribution to values
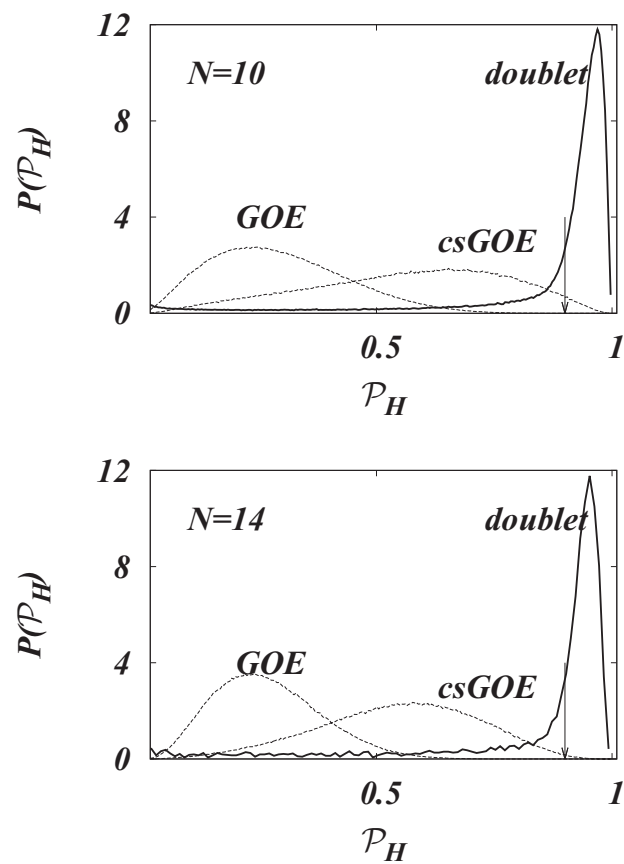

FIG. 7. Distribution of the transfer efficiency $\mathcal{P}_{H}$, eq. (2), for variable network size $N$ and three Hamiltonian ensembles: GOE, GOE with centrosymmetry, and GOE with centrosymmetry and dominant doublet. $\mathcal{P}_{H}=2 \alpha-1$ is indicated by the arrow. The control parameters in (1) and (11) are set to $\xi=2$ and $\alpha=0.95$. 


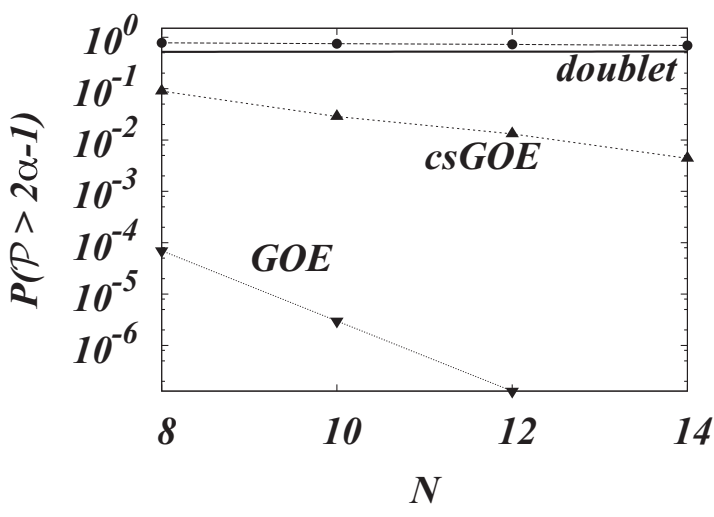

FIG. 8. Density $P\left(\mathcal{P}_{H}>2 \alpha-1\right)$ of efficient network realizations, as a function of the network size $N$, for three different ensembles (GOE, GOE with centrosymmetry, GOE with centrosymmetry and dominant doublet). The theoretical curve for $P\left(t<T_{R}\right)$ (solid line), Eq. (26), forms a lower bound to the dominant doublet ensemble, as expected (see text). The GOE curve is cut off at $N=12$, since it takes too long to sample a sufficient amount of data for larger values of $N$. The control parameters in (1) and (11) are $\alpha=0.95$ and $\xi=2$.

close to one. This is unambiguously achieved by the dominant doublet constraint (a generalization of the CAT mechanism) and in perfect agreement with our predictions.

As for those incidents in Fig. 7 where $\mathcal{P}_{H}<2 \alpha-1$, despite the presence of a dominant doublet, these typically are due to network conformations where $T_{R}<t$, i.e., where the transport is too slow to be efficient. On the other hand, there are also some realizations (such as shown in Fig. 2) where $\mathcal{P}_{H}>2 \alpha-1$ even though $T_{R}<t$. As a matter of fact, there is no obvious one-to-one relation between the first passage time distribution and the efficiency distribution what, however, leaves our overall picture of the transport mechanism fully intact. Also, as $N$ is increased, it might seem that more structure emerges in the region $\mathcal{P}_{H}<2 \alpha-1$. This, however, is just statistical noise: Because of the postselection, together with the strongly decreasing density of dominant doublets in the ensemble (Appendix B), it is difficult to acquire a lot of statistics for $N=14$.

Let us finally extract from Fig. 7 the probability to achieve transfer efficiencies $\mathcal{P}_{H}>2 \alpha-1$, which is simply done by integrating over the corresponding interval of the histograms, for the different ensembles considered. The result displayed in Fig. 8 is yet another impressive demonstration of the effectiveness of centrosymmetry and dominant doublet as robust design principles. Also note that the result for the dominant doublet ensemble confirms the estimate (26): Since $T_{R} / t>1$ guarantees $\mathcal{P}_{H}>2 \alpha-1$, while the inverse is not true (remember Fig. 2), (26) defines a lower bound for $P\left(\mathcal{P}_{H}>2 \alpha-1\right)$, as nicely spelled out by the comparison in Fig. 8 .

\section{DISCUSSION AND CONCLUSIONS}

We described a general mechanism that gives rise to fast and efficient quantum transport on finite, disordered networks. The mechanism rests on two crucial ingredients: The first is the centrosymmetry of the underlying Hamiltonian, which renders the Hamiltonian block-diagonal in the eigenbasis of the exchange matrix, the symmetry operator. The second ingredient is a dominant doublet, that ensures a firm control of the transport properties' statistics, under the coupling to random (or chaotic; recall the original motivation of the CAT mechanism [47]) states which assist the transport. The statistics of the transfer efficiencies and times as shown in Figs. 5 and 7 depend only on the intermediate network sites' density of states $\xi$, and on the average coupling strength $\overline{\|\mathcal{V}\|^{2}}$ of the in- and output sites to the network. These are macroscopically controllable parameters. On the one hand, this means that coherence effects survive simply by stabilising these properties of the ensemble. On the other hand, if such stabilization is possible, one could also imagine controlling transport properties according to the specific needs, simply by controlling these ensemble properties such as the density of states and the typical coupling to the intermediate sites.

The key point of our contribution is to treat near-optimal transport in a context of disorder physics, where we do not strive to avoid disorder altogether, but rather incorporate it in a constructive way. By no means do we wish to control as many degrees of freedom as possible, as hardwired smallscale structures are unavoidably perturbed by omnipresent fluctuations. Rather we provide a framework that optimally controls few coarse grained quantities, only constrained by the above design principles, whereas microscopic details may remain subject to disorder or fluctuations. Our handle of control tunes the statistical properties of the transfer efficiency in the sense that it controls the shape of the distributions in Figs. 5 and 7. Moreover, the transfer time distribution, Fig. 5, is a Cauchy distribution, which, as its possibly most important feature, has an algebraic (fat) tail, guaranteeing that transfer times which are shorter than the Rabi time occur in a relatively large fraction of network realizations. In particular, there is a non-negligible probability for dramatic speed-up (by more than an order of magnitude) of the excitation transfer.

Recently other works concerning ensemble approaches to efficient transport in complex quantum systems have been presented [27,64], where, by randomly sampling networks of dipoles, realizations leading to efficient transfer are identified. When analyzing these networks, one mainly encounters centrosymmetric structures [64]. As these efficient realizations are further investigated, Refs. [27,64] find that typically only a subset of the network sites are significantly populated during the transport, which is a consequence of Hamiltonian eigenvector localization on these network sites. Although Refs. [27,64] encounter different possible backbone structures, typically containing four sites or more, these results are strongly reminiscent of our dominant doublet. In other words, one might say that the dominant doublet is a specific, and (see above) analytically tractable, type of backbone structure. We expect that the more complex backbones of $[27,64]$ can be incorporated into a framework similar to the one which is presented in our present contribution, by adopting models comparable to what is known as resonance-assisted tunnelling in the quantum chaos literature $[65,66]$.

Finally, even though our work is originally inspired by recent developments in photobiology, as we stressed in detail in Refs. [18,32], one might think of various other fields 
where ensemble approaches to quantum transport are rapidly gaining relevance. More specifically, the realm of quantum computation harbours several ideas that relate computational problems to quantum walks $[6,36,67]$, thus relating quantum computation to complex networks. On the other hand, random matrix models have been successfully applied in the study of adiabatic quantum computation [68]. More recently, in the broad discussion on quantum effects in D-Wave Two [69], it became clear that random fluctuations and disorder effects must be incorporated in the study of quantum effects in such real systems [70]. We trust that a model as ours, in all its generality, may also enrich this field.

\section{ACKNOWLEDGMENTS}

R.M. acknowledges support by the Alexander von Humboldt Stiftung. M.W. and A.B. are grateful for funding within the DFG Research Unit 760 and for support through the EU COST Action MP1006. M.W. acknowledges partial funding by the Belgian Inter-university Attraction Poles Programme P6/02 and Fonds Wetenschappelijk Onderzoek (FWO) Vlaanderen Project No. G040710N, and by the German National Academic Foundation.

\section{APPENDIX A: BLOCK DIAGONALIZATION OF $H$}

The procedure to obtain the block diagonal form is the same as in Ref. [39]. We consider $H \in \mathbb{C}^{N \times N}$ to be a symmetric and centrosymmetric matrix. We now represent $H$ in terms of four matrices $A, B, C, D \in \mathbb{C}^{N / 2 \times N / 2}$, such that

$$
H=\left(\begin{array}{ll}
A & B \\
C & D
\end{array}\right) .
$$

This can of course be done for any matrix. Symmetry and centrosymmetry now imply that

$$
B=J^{\prime} C J^{\prime} \text { and } D=J^{\prime} A J^{\prime} .
$$

Here $J^{\prime}$ is an $N / 2 \times N / 2$ matrix such that the exchange operator $J$ is given by

$$
J=\left(\begin{array}{ll}
0 & J^{\prime} \\
J^{\prime} & 0
\end{array}\right) .
$$

Next, a transformation $K$ is defined such that $K \in \mathbb{C}^{N \times N}$ is orthogonal and given by

$$
K=\frac{1}{\sqrt{2}}\left(\begin{array}{cc}
\mathbb{1}_{\frac{N}{2}} & -J^{\prime} \\
\mathbb{1}_{\frac{N}{2}} & J^{\prime}
\end{array}\right) .
$$

Therefore it follows from a simple calculation that

$$
K H K^{T}=\left(\begin{array}{cc}
A-J^{\prime} C & 0 \\
0 & A+J^{\prime} C
\end{array}\right) .
$$

From this result it is now easy to identify $H^{ \pm}=A \pm J^{\prime} C$ in terms of random matrix quantities: Consider that, since $A_{i j},\left(J^{\prime} C\right)_{i j} \sim \mathcal{N}\left(0, \frac{\xi^{2}}{N}\right)$, if $i \neq j$, it follows from basic probability theory that

$$
\left(A+J^{\prime} C\right)_{i j}=A_{i j}+J^{\prime} C_{i j} \sim \mathcal{N}\left(0, \frac{2 \xi^{2}}{N}\right), \quad i \neq j .
$$

Likewise we have

$$
\left(A+J^{\prime} C\right)_{i i}=A_{i i}+J^{\prime} C_{i i} \sim \mathcal{N}\left(0, \frac{4 \xi^{2}}{N}\right) .
$$

Since the sum of two symmetric matrices is again symmetric, and since the components are sampled from a Gaussian distribution, these matrices belong to the GOE. In the case of $A-J^{\prime} C$, there is an extra subtlety because of the minus sign. Here we explicitly use that a Gaussian distribution is symmetric, such that, if $\left(J^{\prime} C\right)_{i j} \sim \mathcal{N}\left(0, \xi^{2} / N\right)$, also $-\left(J^{\prime} C\right)_{i j} \sim$ $\mathcal{N}\left(0, \xi^{2} / N\right)$, which implies

$$
\left(A-J^{\prime} C\right)_{i j} \sim\left\{\begin{array}{lll}
\mathcal{N}\left(0, \frac{2 \xi^{2}}{N}\right), & \text { if } & i \neq j . \\
\mathcal{N}\left(0, \frac{4 \xi^{2}}{N}\right), & \text { if } & i=j .
\end{array}\right.
$$

Consequently, also $A-J^{\prime} C$ is an $N / 2 \times N / 2$ GOE matrix.

\section{APPENDIX B: FINDING DOMINANT DOUBLETS}

In Sec. II, we introduced the dominant doublet as a constraint. From a theoretical point of view, it is also interesting to have an idea of the probability that this constraint holds for any GOE matrix with the centrosymmetry constraint.

At first we quote an interesting result from Ref. [44], which concerns eigenvectors. We consider an $N \times N$ matrix in the GOE. Without loss of generality, the eigenvectors of a GOE matrix can be taken to be real. Since an eigenvector $|\eta\rangle$ can be mapped onto any other real vector by an orthogonal transformation, every eigenvector occurs with the same probability. The only property that needs to be fixed is the norm. This implies that

$$
P_{G O E}(|\eta\rangle)=C \delta\left(1-\sum_{i=1}^{N} \eta_{i}^{2}\right) .
$$

Here $C$ is a normalization factor. After determining $C$ and integrating out $N-1$ components, we obtain the distribution for $y=\eta_{j}^{2}$, where $\eta_{j}$ is just some component of the eigenvector $|\eta\rangle$. The result is given by

$$
\begin{aligned}
P_{G O E}(y) & =\int_{\mathbb{R}^{N}} \prod_{j=1}^{N} d \eta_{i} \delta\left(y-\eta_{1}^{2}\right) P_{G O E}(|\eta\rangle) \\
& =\frac{1}{\sqrt{\pi}} \frac{\Gamma\left(\frac{N}{2}\right)}{\Gamma\left(\frac{N-1}{2}\right)} \frac{(1-y)^{\frac{N-3}{2}}}{\sqrt{y}} . \\
& =\frac{1}{B\left(\frac{1}{2}, \frac{N}{2}-\frac{1}{2}\right)} y^{1 / 2-1}(1-y)^{N / 2-1 / 2-1} .
\end{aligned}
$$

The last step rewrites this function such that $B(a, b)$ denotes a Beta function [62]. This implies that the $y$ follow a Beta distribution,

$$
y \sim \operatorname{Beta}\left(\frac{1}{2}, \frac{N}{2}-\frac{1}{2}\right) .
$$

The quantity of interest is the probability that $|+\rangle$ and $|-\rangle$ from (5) form a dominant doublet. In mathematical terms, this is the probability that for both, $\mathrm{H}^{+}$and $H^{-}$from (4), there exists and eigenvector, denoted $|\tilde{+}\rangle$ and $|\tilde{-}\rangle$, respectively, such that

$$
|\langle\tilde{ \pm}, \pm\rangle|^{2}>\alpha .
$$


This quantity is equivalent to defining

$$
y=\min \left(\max _{i}\left|\left\langle\eta_{i},+\right\rangle\right|^{2}, \max _{i}\left|\left\langle\eta_{i},-\right\rangle\right|^{2}\right),
$$

where $\left\{\left|\eta_{i}\right\rangle\right\}$ denotes the set of eigenvectors of $H$. Eventually, $\left\langle\eta_{i},+\right\rangle$ and $\left\langle\eta_{i},-\right\rangle$ in (B5) are just components of the eigenvector in the eigenbasis of $J$. Remembering (B3), we know that for GOE matrices, these components are distributed according to a Beta distribution. As currently we consider Hamiltonians of the form (4), we have to treat $H^{+}$and $H^{-}$as two independent GOE matrices. This implies that the probability that a component $y_{i}$ is smaller than $\alpha$ is given by

$$
P\left(\left|\left\langle\eta_{i}, \pm\right\rangle\right|^{2} \leqslant \alpha\right)=I_{\alpha}\left(\frac{1}{2}, \frac{N}{4}-\frac{1}{2}\right),
$$

where $I_{\alpha}$ denotes the regularized Beta function [62]. Since our interest lies in the maximum of $\left|\left\langle\eta_{i},+\right\rangle\right|^{2}$, we can simply follow an approach similar to the one presented in Sec. III D, to obtain

$$
\begin{aligned}
P\left(\max _{i}\left|\left\langle\eta_{i}, \pm\right\rangle\right|^{2}>\alpha\right) & =1-\prod_{i} P\left(\left|\left\langle\eta_{i}, \pm\right\rangle\right|_{i}^{2} \leqslant \alpha\right) \\
& =1-\left[I_{\alpha}\left(\frac{1}{2}, \frac{N}{4}-\frac{1}{2}\right)\right]^{N / 2} .
\end{aligned}
$$

Now that we know the probability for both $\max _{i}\left|\left\langle\eta_{i},+\right\rangle\right|^{2}>$ $\alpha$ and $\max _{i}\left|\left\langle\eta_{i},-\right\rangle\right|^{2}>\alpha$, the next step is obtaining the probability that $y=\min \left(\max _{i}\left|\left\langle\eta_{i},+\right\rangle\right|^{2}, \max _{i}\left|\left\langle\eta_{i},-\right\rangle\right|^{2}\right)>\alpha$. In other words, we need the probability that $\max _{i}\left|\left\langle\eta_{i},+\right\rangle\right|^{2}$ and $\max _{i}\left|\left\langle\eta_{i},-\right\rangle\right|^{2}$ are simultaneously larger than $\alpha$. We obtain this probability as

$$
\begin{aligned}
P(y>\alpha) & =P\left(\max _{i}\left|\left\langle\eta_{i},+\right\rangle\right|^{2}>\alpha\right) P\left(\max _{i}\left|\left\langle\eta_{i},-\right\rangle\right|^{2}>\alpha\right) \\
& =\left\{1-\left[I_{\alpha}\left(\frac{1}{2}, \frac{N}{4}-\frac{1}{2}\right)\right]^{N / 2}\right\}^{2} .
\end{aligned}
$$

The resulting distribution (B8) suggests that the probability of finding a dominant doublet Hamiltonian $H$ in the centrosymmetric GOE strongly decreases with the system size $N$.
[1] N. W. Ashcroft and N. D. Mermin, Solid State Physics (Saunders College Publishing, Philadelphia, 1988).

[2] F. Dubin, R. Melet, T. Barisien, R. Grousson, L. Legrand, M. Schott, and V. Voliotis, Nat. Phys. 2, 32 (2006).

[3] Y. Imry, Introduction to Mesoscopic Physics (Oxford University Press, New York, 1997).

[4] T. Kottos and U. Smilansky, Ann. Phys. 274, 76 (1999).

[5] J. Madroñero, A. Ponomarev, A. R. Carvalho, S. Wimberger, C. Viviescas, A. Kolovsky, K. Hornberger, P. Schlagheck, A. Krug, A. Buchleitner, G. Rempe, and M. O. Scully, Adv. At. Mol. Opt. Phys. 53, 33 (2006).

[6] B. Hein and G. Tanner, Phys. Rev. Lett. 103, 260501 (2009).

[7] M. Christandl, N. Datta, A. Ekert, and A. J. Landahl, Phys. Rev. Lett. 92, 187902 (2004).

[8] D. S. Wiersma, P. Bartolini, A. Lagendijk, and R. Righini, Nature (London) 390, 671 (1997).

[9] G. Labeyrie, F. de Tomasi, J.-C. Bernard, C. A. Müller, C. Miniatura, and R. Kaiser, Phys. Rev. Lett. 83, 5266 (1999).

[10] T. Wellens, Appl. Phys. B 95, 189 (2009).

[11] M. Hartung, T. Wellens, C. A. Müller, K. Richter, and P. Schlagheck, Phys. Rev. Lett. 101, 020603 (2008).

[12] F. Jörder, K. Zimmermann, A. Rodriguez, and A. Buchleitner, Phys. Rev. Lett. 113, 063004 (2014).

[13] G. Modugno, Rep. Prog. Phys. 73, 102401 (2010).

[14] S. Wimberger, I. Guarneri, and S. Fishman, Nonlinearity 16, 1381 (2003).

[15] E. Collini and G. D. Scholes, J. Phys. Chem. A 113, 4223 (2009).

[16] G. S. Engel, T. R. Calhoun, E. L. Read, T.-K. Ahn, T. Mancal, Y.-C. Cheng, R. E. Blankenship, and G. R. Fleming, Nature (London) 446, 782 (2007).

[17] T. Mančal, N. Christensson, V. Lukeš, F. Milota, O. Bixner, H. F. Kauffmann, and J. Hauer, J. Phys. Chem. Lett. 3, 1497 (2012).

[18] M. Walschaers, J. Fernandez-de-Cossio Diaz, R. Mulet, and A. Buchleitner, Phys. Rev. Lett. 111, 180601 (2013).
[19] T. Ritz, R. Wiltschko, P. Hore, C. Rodgers, K. Stapput, P. Thalau, C. Timmel, and W. Wiltschko, Biophys. J. 96, 3451 (2009).

[20] G. Scholes, T. Mirkovic, D. Turner, F. Fassioli, and A. Buchleitner, Energy Environ. Sci. 5, 9374 (2012).

[21] M. Hercher, Appl. Opt. 7, 951 (1968).

[22] E. Abrahams, P. W. Anderson, D. C. Licciardello, and T. V. Ramakrishnan, Phys. Rev. Lett. 42, 673 (1979).

[23] P. W. Anderson, Phys. Rev. 109, 1492 (1958).

[24] T. Scholak, F. Mintert, T. Wellens, and A. Buchleitner, Semicond. Semimet. 83, 1 (2010).

[25] T. P. J. Krüger, C. Ilioaia, M. P. Johnson, E. Belgio, P. Horton, A. V. Ruban, and R. van Grondelle, Biophys. J. 105, 1018 (2013).

[26] R. Hildner, D. Brinks, J. B. Nieder, R. J. Cogdell, and N. F. van Hulst, Science 340, 1448 (2013).

[27] S. Mostarda, F. Levi, D. Prada-Gracia, F. Mintert, and F. Rao, Nat. Commun. 4, 2296 (2013).

[28] M. B. Plenio and S. F. Huelga, New J. Phys. 10, 113019 (2008).

[29] M. Mohseni, P. Rebentrost, S. Lloyd, and A. Aspuru-Guzik, J. Phys. Chem. Lett. 129, 174106 (2008).

[30] J. Moix, J. Wu, P. Huo, D. Coker, and J. Cao, J. Phys. Chem. Lett. 2, 3045 (2011).

[31] T. Scholak, F. de Melo, T. Wellens, F. Mintert, and A. Buchleitner, Phys. Rev. E 83, 021912 (2011).

[32] T. Zech, R. Mulet, T. Wellens, and A. Buchleitner, New J. Phys. 16, 055002 (2014).

[33] M. L. Mehta, Random Matrices (Elsevier/Academic Press, Amsterdam, 2004).

[34] T. Scholak, T. Wellens, and A. Buchleitner, Phys. Rev. A 90, 063415 (2014).

[35] A. Broadbent and E. Kashefi, Theo. Comp. Sci. 410, 2489 (2009).

[36] A. M. Childs, Phys. Rev. Lett. 102, 180501 (2009).

[37] C. S. Hamilton, R. Kruse, L. Sansoni, C. Silberhorn, and I. Jex, Phys. Rev. Lett. 113, 083602 (2014). 
[38] The requirement that the initial and final states, |in $\rangle$ and $\mid$ out $\rangle$, be localized on individual sites, is, however, not a strictly necessary ingredient for our subsequent conclusions.

[39] A. Cantoni and P. Butler, Lin. Algebra Appl. 13, 275 (1976).

[40] T. Zech, M. Walschaers, T. Scholak, R. Mulet, T. Wellens, and A. Buchleitner, Fluct. Nois. Lett. 12, 1340007 (2013).

[41] O. Bohigas, in Chaos and Quantum Physics, edited by M.-J. Giannoni, A. Voros, and J. Zinn-Justin, Les Houches Lectures, Vol. Session LII (North-Holland, Amsterdam, 1989).

[42] Note that we here employ a reference time which is 10 times larger than in Refs. [24,31]. However, this does not alter the qualitative result; also see Ref. [46].

[43] E. Akkermans and G. Montambaux, Mesoscopic Physics of Electrons and Photons (Cambridge Univ. Press, Cambridge, 2007).

[44] F. Haake, Quantum Signatures of Chaos (Springer-Verslag, Berlin Heidelberg, 2010).

[45] F. Haake and K. Życzkowski, Phys. Rev. A 42, 1013 (1990).

[46] T. Scholak, T. Wellens, and A. Buchleitner, J. of Phys. B: At. Mol. Opt. Phys. 44, 184012 (2011).

[47] S. Tomsovic and D. Ullmo, Phys. Rev. E 50, 145 (1994).

[48] A. Buchleitner, I. Guarneri, and J. Zakrzewski, Europhys. Lett. 44, 162 (1998).

[49] P. Schlagheck and A. Buchleitner, Eur. Phys. J. D 22, 401 (2003).

[50] S. Wimberger and A. Buchleitner, J. Phys. A: Math. Gen. 34, 7181 (2001).

[51] J. Zakrzewski, D. Delande, and A. Buchleitner, Phys. Rev. E 57, 1458 (1998).

[52] J. Madroñero and A. Buchleitner, Phys. Rev. Lett. 95, 263601 (2005).

[53] J. Madroñero, P. Schlagheck, L. Hilico, B. Grémaud, D. Delande, and A. Buchleitner, Europhys. Lett. 70, 183 (2005).

[54] S. M. Falke, C. A. Rozzi, D. Brida, M. Maiuri, M. Amato, E. Sommer, A. De Sio, A. Rubio, G. Cerullo, E. Molinari, and C. Lienau, Science 344, 1001 (2014).
[55] F. Leyvraz and D. Ullmo, J. Phys. A: Math. Gen. 29, 2529 (1996).

[56] G. López, P. A. Mello, and T. H. Seligman, Z. Phys. A 302, 351 (1981).

[57] G. Ergün and Y. V. Fyodorov, Phys. Rev. E 68, 046124 (2003).

[58] E. F. Fama and R. Roll, J. Am. Stat. Assoc. 63, 817 (1968).

[59] H. S. Seung, H. Sompolinsky, and N. Tishby, Phys. Rev. A 45, 6056 (1992)

[60] L. de Haan and A. Ferreira, Extreme Value Theory: An Introduction, Springer Series in Operations Research and Financial Engineering (Springer, New York, 2007).

[61] F. C. Leone, L. S. Nelson, and R. B. Nottingham, Technometrics 3, 543 (1961).

[62] M. Abramowitz and I. Stegan, Handbook of Mathematical Functions (Dover Publications, New York, 1965).

[63] P. S. Laplace, Stat. Sci. 1, 364 (1986).

[64] S. Mostarda, F. Levi, D. Prada-Gracia, F. Mintert, and F. Rao, arXiv:1312.1833.

[65] O. Brodier, P. Schlagheck, and D. Ullmo, Phys. Rev. Lett. 87, 064101 (2001).

[66] O. Brodier, P. Schlagheck, and D. Ullmo, Ann. Phys. 300, 88 (2002).

[67] E. Farhi and S. Gutmann, Phys. Rev. A 58, 915 (1998).

[68] J. Roland and N. J. Cerf, Phys. Rev. A 71, 032330 (2005).

[69] P. I. Bunyk, E. M. Hoskinson, M. W. Johnson, E. Tolkacheva, F. Altomare, A. J. Berkley, R. Harris, J. P. Hilton, T. Lanting, A. J. Przybysz, and J. Whittaker, IEEE Trans. Appl. Superconductivity 24, 1700110 (2014).

[70] T. Lanting, A. J. Przybysz, A. Y. Smirnov, F. M. Spedalieri, M. H. Amin, A. J. Berkley, R. Harris, F. Altomare, S. Boixo, P. Bunyk, N. Dickson, C. Enderud, J. P. Hilton, E. Hoskinson, M. W. Johnson, E. Ladizinsky, N. Ladizinsky, R. Neufeld, T. Oh, I. Perminov, C. Rich, M. C. Thom, E. Tolkacheva, S. Uchaikin, A. B. Wilson, and G. Rose, Phys. Rev. X 4, 021041 (2014). 